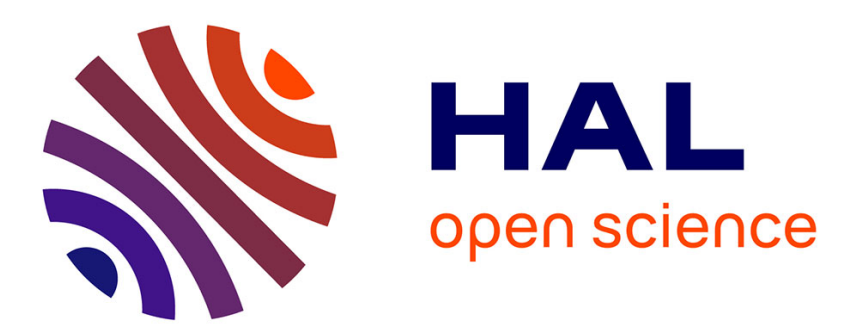

\title{
A Model of Dynamics Driven by Repeated Harmonic Interactions
}

Hiroshi Tamura, Valentin Zagrebnov

\section{To cite this version:}

Hiroshi Tamura, Valentin Zagrebnov. A Model of Dynamics Driven by Repeated Harmonic Interactions. Theoretical and Mathematical Physics, 2016, 187 (3), pp.909-934. 10.1134/S004057791606009X . hal-01176028

\section{HAL Id: hal-01176028 \\ https://hal.science/hal-01176028}

Submitted on 15 Jul 2015

HAL is a multi-disciplinary open access archive for the deposit and dissemination of scientific research documents, whether they are published or not. The documents may come from teaching and research institutions in France or abroad, or from public or private research centers.
L'archive ouverte pluridisciplinaire HAL, est destinée au dépôt et à la diffusion de documents scientifiques de niveau recherche, publiés ou non, émanant des établissements d'enseignement et de recherche français ou étrangers, des laboratoires publics ou privés. 


\title{
A Model of Dynamics Driven by Repeated Harmonic Interactions
}

\author{
Hiroshi Tamura ${ }^{1}$ \\ Institute of Science and Engineering \\ and \\ Graduate School of the Natural Science and Technology \\ Kanazawa University, \\ Kanazawa 920-1192, Japan \\ Valentin A.Zagrebnov ${ }^{2}$ \\ Institut de Mathématiques de Marseille - UMR 7373 \\ CMI-AMU, Technopôle Château-Gombert \\ 39, rue F. Joliot Curie, 13453 Marseille Cedex 13, France \\ and \\ Département de Mathématiques \\ Université d'Aix-Marseille - Luminy, Case 901 \\ 163 av.de Luminy, 13288 Marseille Cedex 09, France
}

\begin{abstract}
We consider an exactly soluble $W^{*}$-dynamical system driven by repeated harmonic interactions. Although dynamics is Hamiltonian and quasi-free, it leads in the large-time limit to relaxation of initial states to a steady state. We found explicitly the rate of the entropy production which accompanies this relaxation. Besides, we study evolution of subsystems to elucidate their eventual correlations and convergence to equilibrium states. Finally we prove a universality of dynamics driven by repeated harmonic perturbations in a short-time interaction limit.
\end{abstract}

\footnotetext{
${ }^{1}$ tamurah@staff.kanazawa-u.ac.jp

${ }^{2}$ Valentin.Zagrebnov@univ-amu.fr
} 


\section{Contents}

1 Preliminaries and the Model 2

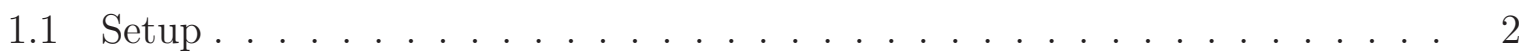

1.2 The Model . . . . . . . . . . . . . . . . . . . 3

2 Hamiltonian Dynamics $\quad 6$

3 Entropy of Quasi-Free States on CCR $C^{*}$-Algebras 10

4 Repeated Perturbations and Entropy Production 13

5 Evolution of Subsystems $\quad 15$

5.1 Convergence to Equilibrium . . . . . . . . . . . . . . . 15

5.2 A Short-Time Limit for Repeated Perturbations . . . . . . . . . . . . . . 22

\section{Preliminaries and the Model}

\subsection{Setup}

We consider a quantum system recently proposed in [TZ]. It is a harmonic system (onemode quantum oscillator $\mathcal{S}$ ) successively perturbed by time-dependent stationary repeated harmonic interactions. This sequence of perturbation is switched on at the moment $t=0$ and it acts successively on the interval $0 \leq t<\infty$. It is a common fashion to present this sequence as repeated interactions of the system $\mathcal{S}$ with an infinite time-equidistant chain: $\mathcal{C}=\mathcal{S}_{1}+\mathcal{S}_{2}+\ldots$, of subsystems $\left\{\mathcal{S}_{k}\right\}_{k \geq 1}$.

Note that there is a physical interpretation [NVZ], [BJM], behind of this mathematical setting. For the model [TZ], the system $\mathcal{C}_{N}$ is identified with a chain of $N$ quantum particles ("atoms") with infinitely many harmonic internal degrees of freedom. They interact one-by-one with a one-mode quantum resonator (cavity) $\mathcal{S}$. This is a caricature of the one-atom maser system. In contract to [NVZ], but similar to the two-level JaynesCummings atoms [BJM], the interaction with harmonic atoms is inelastic. This yields a very different evolution of the model [TZ] comparing to the model [NVZ], in which interaction is completely elastic, see Sections 4 and 5 .

Below we suppose that the states of $\mathcal{S}$ and of every $\mathcal{S}_{k}$ are normal, i.e. defined by the density matrices $\rho_{0}$ and $\left\{\rho_{k}\right\}_{k=1}^{\infty}$ on the Hilbert spaces $\mathscr{H}_{\mathcal{S}}$ and $\left\{\mathscr{H}_{\mathcal{S}_{k}}\right\}_{k=1}^{\infty}$, respectively. The Hilbert space of the total system is then the tensor product $\mathscr{H}_{\mathcal{S}} \otimes \mathscr{H}_{\mathcal{C}}$. Here the infinite product $\mathscr{H}_{\mathcal{C}}=\otimes_{k \geq 1} \mathscr{H}_{\mathcal{S}_{k}}$ stays for the Hilbert space chain.

Since for any fixed moment $t \geq 0$, only a finite number $N(t)$ of repeated interactions are involved into the dynamics, the subsystems $\left\{\mathcal{S}_{k}\right\}_{k>N(t)}$ are still independent for different $k$, as well as they are independent of components $\mathcal{S}$ and $\left\{\mathcal{S}_{k}\right\}_{k=1}^{N(t)}$. On the other hand, the problem of correlations between components $\mathcal{S}, \mathcal{S}_{k}$ for $k \leq N(t)$ and between $\mathcal{S}_{k}, \mathcal{S}_{k^{\prime}}$ for $1 \leq k<k^{\prime} \leq N(t)$ is considered in Section 5 . This peculiarity of the repeated interactions allows to reduce the analysis of dynamics to the finite tensor product: $\mathscr{H}_{\mathcal{C}_{N}}=\otimes_{k=1}^{N} \mathscr{H}_{\mathcal{S}_{k}}$. Then one recovers the above infinite chain $\mathcal{S}+\mathcal{C}$ as the limit $N \rightarrow \infty, a$ 
posteriori. Details of dynamics are presented in the next Section 2. We finish this section by formulation of some of our hypothesises.

Hypothesis (H1): (Initial states) For $t \leq 0$, all components of $\mathcal{S}$ and $\left\{\mathcal{S}_{k}\right\}_{k=1}^{N}$ are independent, i.e. the state of $\mathcal{S}+\mathcal{C}_{N}$ is described as a finite tensor product: $\omega_{\mathcal{S}+\mathcal{C}_{N}}:=$ $\omega_{\mathcal{S}} \otimes \bigotimes_{k=1}^{N} \omega_{\mathcal{S}_{k}}$. We suppose that each of the state in the product is normal.

Hypothesis (H2): (Tuned interaction) We consider repeated perturbations in the tuned regime: for any moment $t \geq 0$ exactly one subsystem ("atom") $\mathcal{S}_{n}$ is interacting with the system $\mathcal{S}$ (quantum resonator) during a fixed time $\tau>0$. Here $n=[t / \tau]+1$, where $[x]$ denotes the integer part of $x \geq 0$.

Let $\mathscr{H}_{0}$ be the Hilbert space for the system $\mathcal{S}$ and $\mathscr{H}_{k}$ be the Hilbert space for the the system $\mathcal{S}_{k}$ for $k=1, \cdots, N$. Then for $k=0,1, \cdots, N$, the space $\mathscr{H}_{k}$ is a copy of the one-mode boson Fock space $\mathscr{F}$ with the vacuum vector $\Omega \in \mathscr{F}$ and with densely defined boson annihilation and (adjoint) creation operators: $a$ and $a^{*}$, defined by $a \Omega=0$. The total system $\mathcal{S}+\mathcal{C}_{N}$ lives in the Hilbert space

$$
\mathscr{H}^{(N)}:=\mathscr{H}_{0} \otimes \bigotimes_{k=1}^{N} \mathscr{H}_{k}=\mathscr{F}^{\otimes(N+1)}
$$

Here $\mathbb{1}$ is the unit operator on $\mathscr{F}$. In the space (1.1) we define operators

$$
b_{k}:=\mathbb{1} \otimes \ldots \otimes \mathbb{1} \otimes a \otimes \mathbb{1} \otimes \ldots \otimes \mathbb{1}, b_{k}^{*}:=\mathbb{1} \otimes \ldots \otimes \mathbb{1} \otimes a^{*} \otimes \mathbb{1} \otimes \ldots \otimes \mathbb{1},
$$

where operator $a$, or $a^{*}$, is the $(k+1)$ th factor in (1.2). Operators (1.2) formally satisfy the Canonical Commutation Relations (CCR)

$$
\left[b_{k}, b_{k^{\prime}}^{*}\right]=\delta_{k, k^{\prime}} \mathbb{1}, \quad\left[b_{k}, b_{k^{\prime}}\right]=\left[b_{k}^{*}, b_{k^{\prime}}^{*}\right]=0, k, k^{\prime}=0,1,2, \cdots, N .
$$

Hypothesis (H3): (Harmonic interaction) The time-dependent repeated interaction described by (H2) is a piecewise constant operator in (1.1). It is the sum over $n \geq 1$ of the bilinear forms in operators (1.2) in the space $\mathscr{H}_{0} \otimes \mathscr{H}_{n}$ :

$$
K_{n}(t):=\chi_{[(n-1) \tau, n \tau)}(t) \eta\left(b_{0}^{*} b_{n}+b_{n}^{*} b_{0}\right), \quad \eta>0 .
$$

Here $\chi_{\mathcal{I}}(x)$ is the characteristic function of the set $\mathcal{I}$.

\subsection{The Model}

For any $N \geq 1$ and $t<N \tau$, the self-adjoint Hamiltonian $H_{N}(t)$ of the non-autonomous system $\mathcal{S}+\mathcal{C}_{N}$ is defined in the space (1.1) as the sum of Hamiltonians corresponding the systems $\mathcal{S}, \mathcal{S}_{k}$ and interaction (1.4) [TZ]:

$$
\begin{aligned}
H_{N}(t) & :=H_{\mathcal{S}}+\sum_{k=1}^{N}\left(H_{\mathcal{S}_{k}}+K_{k}(t)\right) \\
& =E b_{0}^{*} b_{0}+\epsilon \sum_{k=1}^{N} b_{k}^{*} b_{k}+\eta \sum_{k=1}^{N} \chi_{[(k-1) \tau, k \tau)}(t)\left(b_{0}^{*} b_{k}+b_{k}^{*} b_{0}\right),
\end{aligned}
$$


Hypothesis (H4):(Semi-boundedness) To keep the self-adjoint Hamiltonian (1.5) semibounded from below we suppose that $E, \epsilon>0$ and we impose the condition

$$
\eta^{2} \leq E \epsilon
$$

By virtue of (1.4), (1.5) only $\mathcal{S}_{n}$ interacts with $\mathcal{S}$ for $t \in[(n-1) \tau, n \tau), n \geq 1$, i.e. the system $\mathcal{S}+\mathcal{C}_{N}$ is autonomous on this time-interval with self-adjoint Hamiltonian

$$
H_{n}:=E b_{0}^{*} b_{0}+\epsilon \sum_{k=1}^{N} b_{k}^{*} b_{k}+\eta\left(b_{0}^{*} b_{n}+b_{n}^{*} b_{0}\right), n \leq N .
$$

Note that there exists a CCR-preserving linear transformation, which diagonalise (1.7):

$$
\widetilde{H}_{n}:=\varepsilon_{0} c_{0}^{*} c_{0}+\varepsilon_{1} c_{1}^{*} c_{1}+\sum_{k=2}^{N} \varepsilon_{k} c_{k}^{*} c_{k} .
$$

Here $\varepsilon_{2}=\ldots=\varepsilon_{N}=\epsilon$, and $\varepsilon_{0,1}:=\frac{1}{2}\left[(E+\epsilon) \pm \sqrt{(E-\epsilon)^{2}+4 \eta^{2}}\right]>0$ by (H4).

The next key lemma follows from the harmonic structure of the Hamiltonian (1.7).

Lemma 1.1 For $j=0,1,2, \ldots, N$ and $n=1,2, \ldots, N$, one gets

$$
\begin{aligned}
e^{i t H_{n}} b_{j} e^{-i t H_{n}} & =\sum_{k=0}^{N}\left(U_{n}^{*}(t)\right)_{j k} b_{k}, \quad e^{i t H_{n}} b_{j}^{*} e^{-i t H_{n}}=\sum_{k=0}^{N} \overline{\left(U_{n}^{*}(t)\right)_{j k}} b_{k}^{*}, \\
e^{-i t H_{n}} b_{j} e^{i t H_{n}} & =\sum_{k=0}^{N}\left(U_{n}(t)\right)_{j k} b_{k}, \quad e^{-i t H_{n}} b_{j}^{*} e^{i t H_{n}}=\sum_{k=0}^{N} \overline{\left(U_{n}(t)\right)_{j k}} b_{k}^{*},
\end{aligned}
$$

for $t \geq 0$. Here $U_{n}(t)$ and $V_{n}(t)$ are $(N+1) \times(N+1)$ matrices related by $U_{n}(t):=e^{i t \epsilon} V_{n}(t)$, where

$$
\left(V_{n}(t)\right)_{j k}:=\left\{\begin{array}{cl}
g(t) z(t) \delta_{k 0}+g(t) w(t) \delta_{k n} & (j=0) \\
g(t) w(t) \delta_{k 0}+g(t) z(-t) \delta_{k n} & (j=n) \\
\delta_{j k} & \text { (otherwise) }
\end{array}\right.
$$

and

$$
\begin{gathered}
g(t):=e^{i t(E-\epsilon) / 2}, w(t):=\frac{2 i \eta}{\sqrt{(E-\epsilon)^{2}+4 \eta^{2}}} \sin t \sqrt{\frac{(E-\epsilon)^{2}}{4}+\eta^{2}} \\
z(t):=\cos t \sqrt{\frac{(E-\epsilon)^{2}}{4}+\eta^{2}}+\frac{i(E-\epsilon)}{\sqrt{(E-\epsilon)^{2}+4 \eta^{2}}} \sin t \sqrt{\frac{(E-\epsilon)^{2}}{4}+\eta^{2}} .
\end{gathered}
$$

Remark 1.2 Note that by definitions (1.12) and (1.13), we get $|z(t)|^{2}+|w(t)|^{2}=1$, $z(-t)=\overline{z(t)}$ and $w(t)=-\overline{w(t)}$. Therefore, the matrix

$$
M(t):=\left(\begin{array}{cc}
z(t) & w(t) \\
w(t) & z(-t)
\end{array}\right)
$$

is unitary. For $N=1$, one gets $M(t)=\overline{g(t)} V_{1}(t)$, see (1.11). Moreover, (1.9) and (1.10) imply that $\left\{V_{n}(t)\right\}_{t \in \mathbb{R}}$ and $\left\{U_{n}(t)\right\}_{t \in \mathbb{R}}$ are in fact one-parameter groups of $(N+1) \times(N+1)$ unitary matrices. 
Proof (of Lemma 1.1 ): Let $\left\{J_{n}\right\}_{n=1}^{N}$ and $\left\{X_{n}\right\}_{n=1}^{N}$ be $(N+1) \times(N+1)$ Hermitian matrices given by

$$
\begin{aligned}
& \left(J_{n}\right)_{j k}:=\left\{\begin{array}{ll}
1 \quad(j=k=0 & \text { or } j=k=n) \\
0 & \text { otherwise }
\end{array},\right. \\
& \left(X_{n}\right)_{j k}:= \begin{cases}(E-\epsilon) / 2 & (j, k)=(0,0) \\
-(E-\epsilon) / 2 & (j, k)=(n, n) \\
\eta & (j, k)=(0, n) . \\
\eta & (j, k)=(n, 0) \\
0 & \text { otherwise }\end{cases}
\end{aligned}
$$

We define the matrices

$$
Y_{n}:=\epsilon I+\frac{E-\epsilon}{2} J_{n}+X_{n} \quad(n=1, \ldots, N),
$$

where $I$ is the $(N+1) \times(N+1)$ identity matrix. Then Hamiltonian (1.7) takes the form

$$
H_{n}=\sum_{j, k=0}^{N}\left(Y_{n}\right)_{j k} b_{j}^{*} b_{k} .
$$

Since $Y_{n}$ is Hermitian, there exists a diagonal matrix $\Lambda$ and unitary mapping $\mathcal{U}_{n}: \mathbb{R}^{N+1} \rightarrow$ $\mathbb{R}^{N+1}$, such that $Y_{n}=\mathcal{U}_{n}^{*} \Lambda \mathcal{U}_{n}$ holds. Recall that after canonical transformation the matrix $\Lambda:=\left\{\Lambda_{i j}\right\}_{i, j=0}^{N}=\left\{\delta_{i j} \varepsilon_{j}\right\}_{i, j=0}^{N}(1.8)$ is universal and independent of $n$. The new operators:

$$
c_{j}=\sum_{k=0}^{N}\left(\mathcal{U}_{n}\right)_{j k} b_{k}, \quad c_{j}^{*}=\sum_{k=0}^{N} \overline{\left(\mathcal{U}_{n}\right)_{j k}} b_{k}^{*} \quad(j=0,1, \ldots, N),
$$

satisfy CCR in the space $\mathscr{H}^{(N)}(1.1)$ and diagonalise (1.17): $\widetilde{H}_{n}=\sum_{j=0}^{N} \Lambda_{j j} c_{j}^{*} c_{j}$, where $\Lambda_{j j}=\varepsilon_{j}$ (1.8). Therefore, the set of all eigenvectors of $\widetilde{H}_{n}$ is

$$
\left\{\prod_{j=0}^{N} \frac{\left(c_{j}^{*}\right)^{n_{j}}}{\sqrt{n_{j} !}} \Omega \otimes \ldots \otimes \Omega \mid n_{j} \in \mathbb{Z}_{+} \quad(j=0,1, \ldots, N)\right\} .
$$

Note that it forms a complete orthonormal basis in $\mathscr{H}^{(N)}$. The linear envelope $\mathscr{H}_{0}^{(N)}$ of the set (1.19) is invariant subspace for transformations $e^{i t \widetilde{H}_{n}}$ and its norm-closure coincides with $\mathscr{H}^{(N)}$. Then by (1.18) one gets on vectors (1.19):

$$
e^{i t \widetilde{H}_{n}} c_{j} e^{-i t \widetilde{H}_{n}}=e^{-i t \Lambda_{j j}} c_{j}, \quad e^{i t \widetilde{H}_{n}} c_{j}^{*} e^{-i t \widetilde{H}_{n}}=e^{i t \Lambda_{j j}} c_{j}^{*} .
$$

Now taking into account canonical transformation (1.18), we obtain

$$
e^{i t H_{n}} b_{j} e^{-i t H_{n}}=\sum_{k=0}^{N}\left(\mathcal{U}_{n}^{*}\right)_{j k} e^{i t \widetilde{H}_{n}} c_{k} e^{-i t \widetilde{H}_{n}}
$$




$$
=\sum_{k, l=0}^{N}\left(\mathcal{U}_{n}^{*}\right)_{j k} e^{-i t \Lambda_{k k}}\left(\mathcal{U}_{n}\right)_{k l} b_{l}=\sum_{l=0}^{N}\left(e^{-i t \mathcal{U}_{n}^{*} \Lambda \mathcal{U}_{n}}\right)_{j l} b_{l}=\sum_{l=0}^{N}\left(e^{-i t Y_{n}}\right)_{j l} b_{l} .
$$

Similarly we obtain $e^{i t H_{n}} b_{j}^{*} e^{-i t H_{n}}=\sum_{l=0}^{N} \overline{\left(e^{-i t Y_{n}}\right)_{j l}} b_{l}^{*}$.

Note that by virtue of (1.14), (1.15), one has identities

$$
X_{n}^{2}=\left(\frac{(E-\epsilon)^{2}}{4}+\eta^{2}\right) J_{n} \quad \text { and } \quad J_{n} X_{n}=X_{n}
$$

Together with definition (1.16) and (1.11), they yield

$$
\begin{gathered}
e^{i t Y_{n}}=e^{i t \epsilon}\left(I-J_{n}+e^{i t(E-\epsilon) / 2}\left\{J_{n} \cos t \sqrt{\frac{(E-\epsilon)^{2}}{4}+\eta^{2}}\right.\right. \\
\left.\left.+i X_{n}\left[\frac{(E-\epsilon)^{2}}{4}+\eta^{2}\right]^{-1 / 2} \sin t \sqrt{\frac{(E-\epsilon)^{2}}{4}+\eta^{2}}\right\}\right)=e^{i t \epsilon} V_{n}(t)=U_{n}(t) .
\end{gathered}
$$

Inserting now (1.21) into (1.20), we prove (1.9). Since $U_{n}(t)^{*}=U_{n}(-t)$, one can similarly establish (1.10).

Remark 1.3 Hereafter, we are going to use the short-hand notations:

$$
g:=g(\tau), w:=w(\tau), z:=z(\tau) \text { and } V_{n}:=V_{n}(\tau), U_{n}:=U_{n}(\tau)
$$

In Section 2, we give explicit description of the Hamiltonian dynamics for the nonautonomous system $\mathcal{S}+\mathcal{C}$ driven by harmonic repeated interactions (H3). We show that our model of bosons (1.5) is a quasi-free $W^{*}$-dynamical system. In Section 3 we recall formulae for the entropy of the CCR quasi-free states. We use them in Section 4 for calculations of the entropy production. Section 5 is dedicated to analysis of reduced dynamics of subsystems, of their correlations and of convergence to equilibrium. We prove a universality of the short-time interaction limit of this dynamics for the subsystem $\mathcal{S}$.

\section{Hamiltonian Dynamics}

A well-known way to avoid the problem of evolution of unbounded creation-annihilation operators is to construct dynamics of the subsystem $\mathcal{S}$ on the unital Weyl CCR $C^{*}$-algebra $\mathscr{A}(\mathscr{F})$, see e.g. [AJP1] (Lectures 4 and 5), [BR2]. Here $\mathscr{A}(\mathscr{F})$ is generated on the Fock space $\mathscr{F}$ as the operator-norm closure of the linear span $\mathscr{A}_{w}$ of the Weyl operator system:

$$
\left\{\widehat{w}(\alpha)=e^{i \Phi(\alpha) / \sqrt{2}}\right\}_{\alpha \in \mathbb{C}}
$$

Here $\Phi(\alpha):=\bar{\alpha} a+\alpha a^{*}$ is a self-adjoint operator with domain in $\mathscr{F}$ and the CCR take then the Weyl form:

$$
\widehat{w}\left(\alpha_{1}\right) \widehat{w}\left(\alpha_{2}\right)=e^{-i \operatorname{Im}\left(\bar{\alpha}_{1} \alpha_{2}\right) / 2} \widehat{w}\left(\alpha_{1}+\alpha_{2}\right), \quad \alpha_{1}, \alpha_{2} \in \mathbb{C} .
$$


Note that $\mathscr{A}(\mathscr{F})$ is a minimal $C^{*}$-algebra, which contains the linear span $\mathscr{A}_{w}$ of the Weyl operator system (2.1). Algebra $\mathscr{A}(\mathscr{F})$ is contained in the unital $C^{*}$-algebra $\mathcal{L}(\mathscr{F})$ of all bounded operators on $\mathscr{F}$.

Similarly we define the Weyl $C^{*}$-algebra $\mathscr{A}(\mathscr{H}) \subset \mathcal{L}(\mathscr{H})$ over $\mathscr{H}:=\mathscr{H}^{(N)}(1.1)$. It is appropriate for description the system $\mathcal{S}+\mathcal{C}$. This algebra is generated by operators

$$
W(\zeta)=\bigotimes_{k=0}^{N} \widehat{w}\left(\zeta_{k}\right), \quad \zeta=\left\{\zeta_{k}\right\}_{k=0}^{N} \in \mathbb{C}^{N+1}, N \geq 1
$$

Using definitions of the boson operators $\left\{b_{k}, b_{k}^{*}\right\}_{k=1}^{N}$ and of the sesquilinear forms

$$
\langle\zeta, b\rangle:=\sum_{j=0}^{N} \bar{\zeta}_{j} b_{j}, \quad\langle b, \zeta\rangle:=\sum_{j=0}^{N} \zeta_{j} b_{j}^{*}
$$

the Weyl operators (2.3) can be rewritten as

$$
W(\zeta)=\exp [i(\langle\zeta, b\rangle+\langle b, \zeta\rangle) / \sqrt{2}]
$$

We denote by $\mathfrak{C}_{1}(\mathscr{F}) \subset \mathcal{L}(\mathscr{F})$, the set of all trace-class operators on $\mathscr{F}$. A self-adjoint, non-negative operator $\rho \in \mathfrak{C}_{1}(\mathscr{F})$ with unit trace is called density matrix. The state $\omega_{\rho}(\cdot)$ generated on the $C^{*}$-algebra of bounded operators $\mathcal{L}(\mathscr{F})$ by $\rho$ :

$$
\omega_{\rho}(A):=\operatorname{Tr}_{\mathscr{F}}(\rho A), \quad A \in \mathcal{L}(\mathscr{F}),
$$

is a normal state. Let $\left\{\rho_{k}\right\}_{k=0}^{N}$ be density matrices on $\mathscr{F}$. Then the normal product-state on the $C^{*}$-algebra $\mathscr{A}(\mathscr{H})$ (isometrically isomorphic to the tensor product $\otimes_{k=0}^{N} \mathscr{A}(\mathscr{F})$ ) is

$$
\omega_{\rho \otimes}(\cdot):=\operatorname{Tr}_{\mathscr{H}}\left(\rho^{\otimes} \cdot\right), \quad \rho^{\otimes}:=\otimes_{k=0}^{N} \rho_{k} .
$$

If we put $C_{k}(\alpha):=\operatorname{Tr}_{\mathscr{F}}\left[\rho_{k} \widehat{w}(\alpha)\right], \alpha \in \mathbb{C}$, then by (2.3) one obtains for $\rho^{\otimes}(2.7)$ the representation:

$$
\omega_{\rho \otimes}(W(\zeta)):=\operatorname{Tr}_{\mathscr{H}}\left[\rho^{\otimes} W(\zeta)\right]=\prod_{k=0}^{N} C_{k}\left(\zeta_{k}\right) .
$$

Let $\varrho \in \mathfrak{C}_{1}(\mathscr{H})$ be a density matrix on $\mathscr{H}$. Then for the system $\mathcal{S}+\mathcal{C}$, the Hamiltonian evolution $T_{t}: \varrho \mapsto \varrho(t)$ of initial density matrix $\varrho(0):=\varrho$ is defined as a solution of the Cauchy problem for the non-autonomous Liouville equation

$$
\partial_{t} \varrho(t)=L(t)(\varrho(t)),\left.\varrho(t)\right|_{t=0}=\varrho .
$$

By virtue of (1.7) the equation (2.9) is autonomous for each of the interval $[(n-1) \tau, n \tau)$ :

$$
L(t)(\cdot)=L_{n}(\cdot)=-i\left[H_{n}, \cdot\right], \quad t \in[(n-1) \tau, n \tau), n \geqslant 1 .
$$

Since any $t \geqslant 0$ has the representation:

$$
t:=n(t) \tau+\nu(t), n(t):=[t / \tau] \text { and } \nu(t) \in[0, \tau)
$$


by the Markovian independence of generators (2.10), the trace-norm $\left(\|\cdot\|_{1}\right)$-continuous solution of the Cauchy problem (2.9) [Za] takes the iterative form:

$$
\begin{aligned}
& \varrho(t)=T_{t}(\varrho):=T_{\nu(t), n}\left(T_{\tau, n-1}\left(\ldots T_{\tau, 1}(\varrho) \ldots\right)\right)= \\
& e^{-i \nu(t) H_{n}} e^{-i \tau H_{n-1}} \ldots e^{-i \tau H_{1}} \varrho e^{i \tau H_{1}} \ldots e^{i \tau H_{n-1}} e^{i \nu(t) H_{n}} .
\end{aligned}
$$

Here $t \in[(n-1) \tau, n \tau), n=n(t)<N$. By the $\|\cdot\|_{1}$-continuity we obtain from (2.12) that

$$
\varrho(N \tau-0)=\varrho(N \tau)=T_{N \tau}(\varrho)=e^{-i \tau H_{N}} \ldots e^{-i \tau H_{1}} \varrho e^{i \tau H_{1}} \ldots e^{i \tau H_{N}} .
$$

Note that equivalent and often more convenient description of density matrices evolution (2.12) is the dual dynamics $T_{t}^{*}: \mathcal{L}(\mathscr{H}) \rightarrow \mathcal{L}(\mathscr{H}):$

$$
\omega_{T_{t}(\varrho)}(A)=\operatorname{Tr}_{\mathscr{H}}\left(T_{t}(\varrho) A\right)=: \operatorname{Tr}_{\mathscr{H}}\left(\varrho T_{t}^{*}(A)\right), \text { for }(\varrho, A) \in \mathfrak{C}_{1}(\mathscr{F}) \times \mathcal{L}(\mathscr{H}) .
$$

Since $t \mapsto T_{t}(\varrho)$ is $\|\cdot\|_{1}$-continuous and since $\mathcal{L}(\mathscr{H})$ is topologically dual of $\mathfrak{C}_{1}(\mathscr{H})$, one gets that $t \mapsto T_{t}^{*}(A)$ in (2.14) is a one-parameter $*$-automorphism of the unital $C^{*}$-algebra of bounded operators $\mathcal{L}(\mathscr{H})$. The automorphism of the $C^{*}$-dynamical system $\left(\mathcal{L}(\mathscr{H}), T_{t}^{*}\right)$ is not time-continuous for bosons. To ensure the continuity of $T_{t}^{*}$ one considers instead of the $C^{*}$-algebra $\mathscr{A}(\mathscr{H}) \subset \mathcal{L}(\mathscr{H})$, the von Neumann algebra $\mathfrak{M}(\mathscr{H})$, which is closure of the Weyl linear span $\mathscr{A}_{w}$ generated by $(2.1),(2.3)$ in the weak*-topology. Since it is weaker than $C^{*}$-algebra topology, $\mathfrak{M}(\mathscr{H})$ is $*$-isomorphic to $\mathcal{L}(\mathscr{H})$. Then $\|\cdot\|_{1}$-continuity of $T_{t}(\varrho)$ implies continuity of the dual mapping $t \mapsto T_{t}^{*}(A)$ in the weak ${ }^{*}$-topology on $\mathfrak{M}(\mathscr{H})$ and defines a $W^{*}$-dynamical system $\left(\mathfrak{M}(\mathscr{H}), T_{t}^{*}\right)$, see e.g. [AJP1] (Lectures 2 and 4$)$.

Remark 2.1 Below we show that $T_{t}^{*}$ maps $\mathscr{A}(\mathscr{H})$ into itself, and that the action of $T_{t}^{*}$ on Weyl operators can be calculated in the explicit form. Since $\mathscr{A}(\mathscr{H})$ is $*$-weakly dense in $\mathcal{L}(\mathscr{H})$, these allow to deduce properties of evolution $\rho(t)$.

Using (2.13) and dual representation (2.14), we prove the main result of this section.

Lemma 2.2 For $t=N \tau$, the expectation (2.8) of the Weyl operator (2.5) with respect to the evolved state has the form

$$
\omega_{\rho(N \tau)}(W(\zeta))=\omega_{\rho}\left(W\left(U_{1} \ldots U_{N} \zeta\right)\right)=\prod_{k=0}^{N} C_{k}\left(\left(U_{1} \ldots U_{N} \zeta\right)_{k}\right) .
$$

Here

$$
\left(U_{1} \ldots U_{N} \zeta\right)_{0}=e^{i N \tau \epsilon}\left((g z)^{N} \zeta_{0}+\sum_{j=1}^{N} g w(g z)^{j-1} \zeta_{j}\right)
$$

whereas

$$
\left(U_{1} \ldots U_{N} \zeta\right)_{k}=e^{i N \tau \epsilon}\left(g w(g z)^{N-k} \zeta_{0}+g \bar{z} \zeta_{k}+\sum_{j=k+1}^{N} g^{2} w^{2}(g z)^{j-k-1} \zeta_{j}\right),
$$

for $0<k<N$, and

$$
\left(U_{1} \ldots U_{N} \zeta\right)_{N}=e^{i N \tau \epsilon}\left(g w \zeta_{0}+g \bar{z} \zeta_{N}\right),
$$

see definitions (1.12) and (1.13). 
Proof : Note that (2.8), (2.13) and duality (2.14) yield

$$
\begin{aligned}
\omega_{\rho(N \tau)}(W(\zeta)) & =\operatorname{Tr}_{\mathscr{H}}\left[\rho T_{N \tau}^{*}(W(\zeta))\right]=\operatorname{Tr}_{\mathscr{H}}\left[\rho e^{i \tau H_{1}} \ldots e^{i \tau H_{N}} W(\zeta) e^{-i \tau H_{N}} \ldots e^{-i \tau H_{1}}\right] \\
& =\operatorname{Tr}_{\mathscr{H}}\left[\rho W\left(U_{1} \ldots U_{N} \zeta\right)\right]=\prod_{k=0}^{N} C_{k}\left(\left(U_{1} \ldots U_{N} \zeta\right)_{k}\right) .
\end{aligned}
$$

To generate the mapping $\zeta \mapsto U_{1} \ldots U_{N} \zeta$ in (2.19), we use Lemma 1.1 and sesquilinear forms (2.4) to obtain

$$
\begin{gathered}
e^{i \tau H_{1}} \ldots e^{i \tau H_{N}}\langle\zeta, b\rangle e^{-i \tau H_{N}} \ldots e^{-i \tau H_{1}}=\left\langle\zeta, U_{N}^{*} \ldots U_{1}^{*} b\right\rangle \\
=\left\langle U_{1} \ldots U_{N} \zeta, b\right\rangle
\end{gathered}
$$

and the similar expression for its conjugate, which we then insert into (2.5).

Moreover, by the same Lemma 1.1, we get that $U_{1} \ldots U_{N} \zeta=e^{i N \tau \epsilon} V_{1} \ldots V_{N} \zeta$, where

$$
\left(V_{1} \ldots V_{N}\right)_{0 j}= \begin{cases}\left(V_{1}\right)_{00} \ldots\left(V_{N}\right)_{00}=(g z)^{N} & (j=0) \\ \left(V_{1}\right)_{00} \ldots\left(V_{j-1}\right)_{00}\left(V_{j}\right)_{0 j}\left(V_{j+1}\right)_{j j} \ldots\left(V_{N}\right)_{j j}=(g z)^{j-1} g w & (0<j \leqslant N),\end{cases}
$$

and for $0<k \leqslant N$ :

$$
\left(V_{1} \ldots V_{N}\right)_{k j}= \begin{cases}\left(V_{1} \ldots V_{k-1}\right)_{k k}\left(V_{k}\right)_{k 0}\left(V_{k+1} \ldots V_{N}\right)_{00}=g w(g z)^{N-k} & (j=0) \\ 0 & (0<j<k) \\ \left(V_{1} \ldots V_{k-1}\right)_{k k}\left(V_{k}\right)_{k k}\left(V_{k+1} \ldots V_{N}\right)_{k k}=g \bar{z} & (j=k) \\ \left(V_{1} \ldots V_{k-1}\right)_{k k}\left(V_{k}\right)_{k 0}\left(V_{k+1} \ldots V_{j-1}\right)_{00}\left(V_{j}\right)_{0 j}\left(V_{j+1} \ldots V_{N}\right)_{j j} & (k<j \leqslant N) .\end{cases}
$$

Collecting these formulae, one obtains explicit expressions for components (2.16) and (2.17) of the vector $U_{1} \ldots U_{N} \zeta$.

Remark 2.3 Note that for a fixed $N$ and for any $t=m \tau, 1 \leq m \leq N$, the arguments of Lemma 2.2 give a general formula

$$
\begin{aligned}
\omega_{\rho(m \tau)}(W(\zeta)) & =\omega_{\rho}\left(T_{m \tau}^{*}(W(\zeta))\right)=\omega_{\rho}\left(W\left(U_{1} \ldots U_{m} \zeta\right)\right) \\
& =\prod_{k=0}^{N} C_{k}\left(\left(U_{1} \ldots U_{m} \zeta\right)_{k}\right) .
\end{aligned}
$$

Following the same line of reasoning as for (2.17) one obtains explicit formulae for the components $\left\{\left(U_{1} \ldots U_{m} \zeta\right)_{k}\right\}_{k=0}^{N}$ :

$$
\begin{aligned}
& \left(U_{1} \ldots U_{m} \zeta\right)_{k}= \\
& \begin{cases}e^{i m \tau \epsilon}\left((g z)^{m} \zeta_{0}+\sum_{j=1}^{m} g w(g z)^{j-1} \zeta_{j}\right) & (k=0) \\
e^{i m \tau \epsilon}\left(g w(g z)^{m-k} \zeta_{0}+g \bar{z} \zeta_{k}+\sum_{j=k+1}^{m} g^{2} w^{2}(g z)^{j-k-1} \zeta_{j}\right) & (1 \leqslant k<m) \\
e^{i m \tau \epsilon}\left(g w \zeta_{0}+g \bar{z} \zeta_{m}\right) & (k=m) \\
e^{i m \tau \epsilon} \zeta_{k} & (m<k \leqslant N)\end{cases}
\end{aligned}
$$

Note that for $m=N$, these formulae coincide with (2.16)-(2.18), except the last line, which is void in this case. 
Remark 2.4 Recall that unity preserving *-dynamics $t \mapsto T_{t}^{*}$ on the von Neumann algebra $\mathfrak{M}(\mathscr{H})$ generated by $\{W(\zeta)\}_{\zeta \in \mathbb{C}}$ (2.5) is quasi-free, if there exist a mapping $U_{t}: \zeta \mapsto U_{t} \zeta$ and a complex-valued function $\Omega_{t}: \zeta \mapsto \Omega_{t}(\zeta)$, such that

$$
T_{t}^{*}(W(\zeta))=\Omega_{t}(\zeta) W\left(U_{t} \zeta\right), \Omega_{0}=1, U_{0}=I
$$

see e.g., [AJP1], [BR2] or [Ve]. Then by Remark 2.3, the step-wise dynamics

$$
T_{m \tau}^{*}(W(\zeta))=W\left(U_{1} \ldots U_{m} \zeta\right), \quad m=0,1, \ldots, N
$$

is quasi-free, with $\Omega_{t}(\zeta)=1$ and the matrices $\left\{U_{j}\right\}_{j=1}^{N}$ on $\mathbb{C}^{N+1}$ defined by Lemma 1.1 .

\section{Entropy of Quasi-Free States on CCR $C^{*}$-Algebras}

In this section, we establish some useful formulae relating expectations of the Weyl operators (Weyl characteristic function) and the entropy of boson quasi-free states. We formulate them in a way that is restricted but sufficient for our purposes. For general settings see, e.g. [Fa], [AJP1], [BR2], [Ve] and references therein.

Definition 3.1 A state $\omega$ on the $C C R C^{*}$-algebra $\mathscr{A}(\mathscr{F})$ (2.1) is called quasi-free, if its characteristic function has the form

$$
\omega(\widehat{w}(\alpha)):=e^{-\frac{1}{4}|\alpha|^{2}-\frac{1}{2} h(\alpha)},
$$

where $h: \alpha \mapsto \widehat{h}(\alpha, \alpha)$ is a (closable) non-negative sesquilinear form on $\mathbb{C} \times \mathbb{C}$. A quasi-free state $\omega$ is gauge-invariant if $\omega(\widehat{w}(\alpha))=\omega\left(\widehat{w}\left(e^{i \varphi} \alpha\right)\right)$ for $\varphi \in[0,2 \pi)$.

Let $\omega_{\beta}$ denote the Gibbs state with parameter $\beta$ (dimensionless inverse temperature) given by the density matrix $\rho(\beta)=e^{-\beta a^{*} a} / Z(\beta)$, where $Z(\beta)=\left(1-e^{-\beta}\right)^{-1}$. Since

$$
\omega_{\beta}(\widehat{w}(\alpha))=e^{-\frac{1}{4}|\alpha|^{2}-\frac{1}{2} h_{\beta}(\alpha)}, \quad h_{\beta}(\alpha)=\frac{|\alpha|^{2}}{e^{\beta}-1}, \alpha \in \mathbb{C},
$$

this state is quasi-free and gauge-invariant. Note that the entropy of $\omega_{\beta}$ is given by

$$
s(\beta):=-\operatorname{Tr}_{\mathscr{F}}[\rho(\beta) \ln \rho(\beta)]=\beta \omega_{\beta}\left(a^{*} a\right)-\ln \left(1-e^{-\beta}\right) \text { and } \omega_{\beta}\left(a^{*} a\right)=\frac{1}{e^{\beta}-1} .
$$

In terms of the variable $x:=\left(1+e^{-\beta}\right) /\left(1-e^{-\beta}\right)$ the entropy (3.3) is

$$
s(\beta)=\sigma(x):=\frac{x+1}{2} \ln \frac{x+1}{2}-\frac{x-1}{2} \ln \frac{x-1}{2} .
$$

Here $\sigma:(1, \infty) \rightarrow(0, \infty)$ and $\sigma^{\prime}(x)>0$.

To extend (3.4) to the space (1.1) we note that a general gauge-invariant quasi-free states on the CCR $C^{*}$-algebra $\mathscr{A}(\mathscr{H})$ are defined by density matrices of the form [Ve]:

$$
\rho_{L}=\frac{1}{Z_{L}} e^{-\langle b, L b\rangle}, Z_{L}=\operatorname{det}\left[I-e^{-L}\right]^{-1}
$$


Here sesquilinear operator-valued forms $\langle b, L b\rangle=\sum_{n, m=0}^{N} \ell_{n m} b_{n}^{*} b_{m}$ are parameterised by $(N+1) \times(N+1)$ positive-definite Hermitian matrix $L=\left\{\ell_{n m}\right\}_{0 \leqslant n, m \leqslant N}$. Note that the *-automorphism $G_{\varphi}$ on $\mathscr{A}(\mathscr{H})$ (the gauge transformation) :

$$
G_{\varphi}: b_{n}^{*} \mapsto b_{n}^{*} e^{i \varphi}, b_{m} \mapsto b_{m} e^{-i \varphi} \quad(\varphi \in \mathbb{R}, n, m=0,1, \ldots N),
$$

leaves the state (3.5) invariant. Then characteristic function of the Weyl operators $W(\zeta)$ takes the form

$$
\omega_{\rho_{L}}(W(\zeta))=\operatorname{Tr}_{\mathscr{H}}\left[\rho_{L} W(\zeta)\right]=\exp \left[-\frac{1}{4}\langle\zeta, \zeta\rangle-\frac{1}{2}\left\langle\zeta, \frac{I}{e^{L}-I} \zeta\right\rangle\right]
$$

Here the (transposed) vector in the argument is $\zeta^{\operatorname{tr}}=\left(\zeta_{0}, \zeta_{1}, \ldots \zeta_{N}\right) \in \mathbb{C}^{N+1}$. Note that the entropy of the state $\omega_{\rho_{L}}$ is given by

$$
S\left(\rho_{L}\right)=-\operatorname{Tr}_{\mathscr{C}}\left[\rho_{L} \ln \rho_{L}\right]=\operatorname{tr}_{\mathbb{C}^{N+1}}\left[L\left(e^{L}-I\right)^{-1}-\ln \left(I-e^{-L}\right)\right] .
$$

If we define the matrix $X:=\left(I+e^{-L}\right)\left(I-e^{-L}\right)^{-1}$, then the characteristic function (3.7) takes the form:

$$
\omega_{\rho_{L}}(W(\zeta))=\exp \left[-\frac{1}{4}\langle\zeta, X \zeta\rangle\right]
$$

and for the entropy (3.8) we obtain

$$
S\left(\rho_{L}\right)=\operatorname{tr}\left[\frac{X+I}{2} \ln \frac{X+I}{2}-\frac{X-I}{2} \ln \frac{X-I}{2}\right] .
$$

Below we need a bit more specified set up than (3.9), (3.10). Let $\rho(\beta, \delta ; \xi)$ be density matrix of a quasi-free state (3.5) corresponding to the operator-valued sesquilinear form

$$
\langle b, L(\beta, \delta ; \xi) b\rangle:=\beta \sum_{n=0}^{N} b_{n}^{*} b_{n}+\delta\langle b, \xi\rangle\langle\xi, b\rangle .
$$

on $\mathbb{C}^{N+1} \times \mathbb{C}^{N+1}$. Here $\beta>0, \delta>-\beta$, and the vector $\xi^{\text {tr }}=\left(\xi_{0}, \xi_{1}, \ldots, \xi_{N}\right) \in \mathbb{C}^{N+1}$.

Lemma 3.2 The partition function of the state

$$
\rho(\beta, \delta ; \xi)=\frac{1}{Z(\beta, \delta ; \xi)} \exp [-\langle b, L(\beta, \delta ; \xi) b\rangle]
$$

is given by

$$
Z(\beta, \delta ; \xi)=\operatorname{Tr}_{\mathscr{H}}\left[e^{-\langle b, L(\beta, \delta ; \xi) b\rangle}\right]=\left(1-e^{-\beta}\right)^{-N}\left(1-e^{-(\beta+\delta\langle\xi, \xi\rangle)}\right)^{-1} .
$$

The characteristic function and the entropy of this state are respectively:

$$
\begin{gathered}
\operatorname{Tr}_{\mathscr{H}}[\rho(\beta, \delta ; \xi) W(\zeta)]=\exp \left[-\frac{1}{4} \frac{1+e^{-\beta}}{1-e^{-\beta}}\langle\zeta, \zeta\rangle\right] \\
\times \exp \left[-\frac{1}{4}\left(\frac{1+e^{-\beta-\delta\langle\xi, \xi\rangle}}{1-e^{-\beta-\delta\langle\xi, \xi\rangle}}-\frac{1+e^{-\beta}}{1-e^{-\beta}}\right)|\langle\xi, \zeta\rangle|^{2} /\langle\xi, \xi\rangle\right],
\end{gathered}
$$

and

$$
S(\rho(\beta, \delta ; \xi))=-\operatorname{Tr}_{\mathscr{H}}[\rho(\beta, \delta ; \xi) \ln \rho(\beta, \delta ; \xi)]=N s(\beta)+s(\beta+\delta\langle\xi, \xi\rangle) .
$$


Proof: Proof of (3.12) follows from (3.5) and (3.11). Indeed, since by (3.5) any orthogonal transformation $\mathcal{O}$ on $\mathbb{C}^{N+1}$ leaves the partition function invariant: $Z_{\mathcal{O}^{T} L \mathcal{O}}=Z_{L}$, one can calculate it with $O \xi$ (instead of $\xi$ ), where $\mathcal{O} \xi$ has only one non-zero component equals to the vector norm $\langle\xi, \xi\rangle^{1 / 2}$. Then the right-hand side of (3.12) follows straightforwardly from the calculation of the left-hand side for this choice of $\mathcal{O} \xi$.

Since this transformation $\mathcal{O}$ also diagonalise the matrix $L:=L(\beta, \delta ; \xi)$, one uses it to simplify (3.9) and then to return back to $\xi$ at the last step. To this aim we note that

$$
\begin{aligned}
& \omega_{\rho_{L}}(W(\zeta))=\exp \left[-\frac{1}{4}\left\langle\mathcal{O} \zeta, \mathcal{O} X \mathcal{O}^{*} \mathcal{O} \zeta\right\rangle\right]= \\
& \exp \left[-\frac{1}{4} \frac{1+e^{-\beta}}{1-e^{-\beta}}\langle\mathcal{O} \zeta, \mathcal{O} \zeta\rangle^{\prime}\right] \exp \left[-\frac{1}{4} \frac{1+e^{-\beta-\delta\langle\xi, \xi\rangle}}{1-e^{-\beta-\delta\langle\xi, \xi\rangle}}\left|(\mathcal{O} \zeta)_{0}\right|^{2}\right] .
\end{aligned}
$$

Here $\langle\mathcal{O} \zeta, \mathcal{O} \zeta\rangle^{\prime}:=\sum_{k=1}^{N}\left|(\mathcal{O} \zeta)_{k}\right|^{2}$ and we choose transformation $\mathcal{O}$ in such a way that $(\mathcal{O} \xi)_{j}=\delta_{0, j}\|\xi\|$. Since

$$
\left|(\mathcal{O} \zeta)_{0}\right|^{2}=\frac{1}{\langle\xi, \xi\rangle}\langle\mathcal{O} \zeta, \mathcal{O} \xi\rangle\langle\mathcal{O} \xi, \mathcal{O} \zeta\rangle
$$

the identities (3.15) prove (3.13). The same method is valid for entropy (3.8). Calculation of the trace in diagonal representation for $L=L(\beta, \delta ; \xi)$ gives formula (3.14).

Recall that the state $\omega$ on the CCR $C^{*}$-algebra $\mathscr{A}(\mathscr{H})$ is regular, if the map $s \mapsto$ $\omega(W(s \zeta))$ is a continuous function of $s \in \mathbb{R}$ for any $\zeta \in \mathbb{C}^{N+1}$. This property follows from the explicit expression (3.13). Since by the Araki-Segal theorem, see e.g. [AJP1](Lecture $5)$, a regular state is completely defined by its characteristic function, (3.13) and (3.14) yield the following statement.

Lemma 3.3 The entropy $S(\rho)$ of the quasi-free state $\omega_{\rho}$ on the $C C R C^{*}$-algebra $\mathscr{A}(\mathscr{H})$ with characteristic function

$$
\omega_{\rho}(W(\zeta))=\exp \left[-\frac{1}{4}\left(x\langle\zeta, \zeta\rangle+x_{0}|\langle\xi, \zeta\rangle|^{2}\right)\right]
$$

is uniquely determined by the parameters $\left(\xi, x, x_{0}\right)$, where $\xi \in \mathbb{C}^{N+1}$, $x>1, x_{0}>1-x$ and it has the form

$$
S(\rho)=N \sigma(x)+\sigma\left(x+x_{0}\langle\xi, \xi\rangle\right),
$$

where $\sigma(\cdot)$ is defined by (3.4).

Proof: The proof follows directly from definition (3.4), if one puts

$$
x_{0}\langle\xi, \xi\rangle=\frac{1+e^{-\beta-\delta\langle\xi, \xi\rangle}}{1-e^{-\beta-\delta\langle\xi, \xi\rangle}}-\frac{1+e^{-\beta}}{1-e^{-\beta}}
$$

in (3.13) and uses (3.4) in (3.14). 


\section{Repeated Perturbations and Entropy Production}

We consider evolution (2.12) of the system $\mathcal{S}+\mathcal{C}$, when initial density matrix (2.7) corresponds to the product of gauge-invariant Gibbs quasi-free states with parameter $\beta_{0} \geq 0$ for $\mathcal{S}$ and with parameter $\beta \geq 0$ for $\mathcal{C}$ :

$$
\rho=\rho_{0} \otimes \bigotimes_{k=1}^{N} \rho_{k}, \quad \rho_{0}=e^{-\beta_{0} a^{*} a} / Z\left(\beta_{0}\right), \quad \rho_{k}=e^{-\beta a^{*} a} / Z(\beta), k=1,2, \ldots, N .
$$

This case corresponds to $\rho_{L}$ in (3.5) with diagonal matrix $L=\operatorname{diag}\left(\beta_{0}, \beta, \ldots, \beta\right)$ and to $\rho(\beta, \delta ; \xi)$ in representation $(3.11)$ with $(\beta, \delta ; \xi)=\left(\beta, \beta_{0}-\beta ; e\right)$, i.e.,

$$
\rho=\rho\left(\beta, \beta_{0}-\beta ; e\right)=\exp \left[-\beta_{0} b_{0}^{*} b_{0}-\beta \sum_{j=1}^{N} b_{j}^{*} b_{j}\right] / Z\left(\beta, \beta_{0}-\beta\right) .
$$

Here $e^{\operatorname{tr}}=(1,0, \ldots, 0) \in \mathbb{C}^{N+1}$ and

$$
Z\left(\beta, \beta_{0}-\beta\right)=Z\left(\beta_{0}\right) Z(\beta)^{N}=\frac{1}{\left(1-e^{-\beta_{0}}\right)\left(1-e^{-\beta}\right)^{N}} .
$$

A straightforward application of formulae (3.13), (3.14) and Lemma 3.2 for $\xi=e$ (i.e. for $\langle\xi, \xi\rangle=1,\langle\xi, \zeta\rangle=\zeta_{0}$ ) to the state (4.1) (or (4.2)), yields the following statement:

Lemma 4.1 The characteristic function of (4.1) (or (4.2)) is

$$
\begin{aligned}
& \omega_{\rho}(W(\zeta))=\operatorname{Tr}_{\mathscr{H}}[\rho W(\zeta)]= \\
& \exp \left[-\frac{\left|\zeta_{0}\right|^{2}}{4}\left(\frac{1+e^{-\beta_{0}}}{1-e^{-\beta_{0}}}-\frac{1+e^{-\beta}}{1-e^{-\beta}}\right)-\frac{\langle\zeta, \zeta\rangle}{4} \frac{1+e^{-\beta}}{1-e^{-\beta}}\right],
\end{aligned}
$$

and the entropy is equal to

$$
S(\rho)=N s(\beta)+s\left(\beta_{0}\right)
$$

Since by (2.12) the density matrix $\rho(t)$ of the total system $\mathcal{S}+\mathcal{C}$ for $t=N \tau$ is

$$
\rho(N \tau)=e^{-i \tau H_{N}} \ldots e^{-i \tau H_{1}} \rho e^{i \tau H_{1}} \ldots e^{i \tau H_{N}},
$$

we obtain for evolution of the characteristic function and the entropy the statement:

Lemma 4.2 Characteristic function of the state with density matrix (4.5) is equal to

$$
\begin{aligned}
& \omega_{\rho(N \tau)}(W(\zeta))= \\
& \exp \left[-\frac{\left|\left(U_{1} \ldots U_{N} \zeta\right)_{0}\right|^{2}}{4}\left(\frac{1+e^{-\beta_{0}}}{1-e^{-\beta_{0}}}-\frac{1+e^{-\beta}}{1-e^{-\beta}}\right)-\frac{\langle\zeta, \zeta\rangle}{4} \frac{1+e^{-\beta}}{1-e^{-\beta}}\right],
\end{aligned}
$$

whereas the total entropy rests invariant:

$$
S(\rho(N \tau))=S(\rho)=N s(\beta)+s\left(\beta_{0}\right) .
$$

Here the mapping $U_{1} \ldots U_{N}: \mathbb{C}^{N+1} \rightarrow \mathbb{C}^{N+1}$ is given by (2.16) and (2.17). 
Proof: From (2.15), one gets $\omega_{\rho(N \tau)}(W(\zeta))=\omega_{\rho}\left(W\left(U_{1} \ldots U_{N} \zeta\right)\right)$. Since the mappings $U_{j}: \mathbb{C}^{N+1} \rightarrow \mathbb{C}^{N+1}, j=1, \ldots, N$ are unitary (Lemma 2.2), (4.3) yields (4.6). Finally, we obtain that the mapping (4.5) leaves the total entropy (4.4) invariant, see (3.3).

Let $\omega$ and $\omega_{0}$ be two normal states on the Weyl CCR algebra $\mathscr{A}(\mathscr{H})$ with density matrices $\varrho$ and $\varrho_{0}$. Following Araki [Ar1], we introduce the relative entropy of the state $\omega$ with respect to $\omega_{0}$ :

$$
\operatorname{Ent}\left(\varrho \mid \varrho_{0}\right):=\operatorname{Tr}_{\mathscr{H}}\left[\varrho\left(\ln \varrho-\ln \varrho_{0}\right)\right] \geq 0
$$

see also [AJP3].

Lemma 4.3 The relative entropy of $\omega_{\rho(N \tau)}$ with respect to $\omega_{\rho}$ is

$$
\operatorname{Ent}(\rho(N \tau) \mid \rho)=\frac{\left(\beta_{0}-\beta\right)\left(e^{\beta_{0}}-e^{\beta}\right)}{\left(e^{\beta_{0}}-1\right)\left(e^{\beta}-1\right)}\left(1-|z|^{2 N}\right)
$$

where $z:=z(\tau)$ is defined by (1.13) and (1.22).

Proof: The trace cyclicity yields

$$
\begin{gathered}
\operatorname{Ent}(\rho(N \tau) \mid \rho)=\operatorname{Tr}_{\mathscr{H}}[\rho(N \tau)(\ln \rho(N \tau)-\ln \rho)] \\
=\operatorname{Tr}_{\mathscr{H}}\left[\rho\left(\ln \rho-e^{i \tau H_{1}} \ldots e^{i \tau H_{N}} \ln \rho e^{-i \tau H_{N}} \ldots e^{-i \tau H_{1}}\right)\right] \\
=\frac{\beta-\beta_{0}}{Z\left(\beta, \beta_{0}-\beta\right)} \operatorname{Tr}_{\mathscr{H}}\left[e^{-\beta_{0} b_{0}^{*} b_{0}-\beta \sum_{j=1}^{N} b_{j}^{*} b_{j}}\left(b_{0}^{*} b_{0}-e^{i \tau H_{1}} \ldots e^{i \tau H_{N}} b_{0}^{*} b_{0} e^{-i \tau H_{N}} \ldots e^{-i \tau H_{1}}\right)\right] .
\end{gathered}
$$

Note that one gets $b_{0}^{*} b_{0}=\langle b, e\rangle\langle e, b\rangle$ by (2.4). Hence, (2.20) implies

$$
e^{i \tau H_{1}} \cdots e^{i \tau H_{N}} b_{0}^{*} b_{0} e^{-i \tau H_{N}} \cdots e^{-i \tau H_{1}}=\sum_{k=0}^{N}\left(U_{1} \ldots U_{N} e\right)_{k} b_{k}^{*} \sum_{k^{\prime}=0}^{N} \overline{\left(U_{1} \ldots U_{N} e\right)_{k^{\prime}}} b_{k^{\prime}}
$$

Note also that the gauge invariance of the state $\rho$ implies the selection rule:

$$
\frac{1}{Z\left(\beta, \beta_{0}-\beta\right)} \operatorname{Tr}_{\mathscr{H}}\left[e^{-\beta_{0} b_{0}^{*} b_{0}-\beta \sum_{j=1}^{N} b_{j}^{*} b_{j}} b_{k}^{*} b_{k^{\prime}}\right]=0 \text { for } k \neq k^{\prime} .
$$

By this rule after injection of (4.10) into (4.9) only diagonal terms with $k=k^{\prime}$ survive in the expectation:

$$
\begin{aligned}
& \operatorname{Ent}(\rho(N \tau) \mid \rho)= \\
& \frac{\beta-\beta_{0}}{Z\left(\beta, \beta_{0}-\beta\right)} \operatorname{Tr}_{\mathscr{H}}\left[e^{-\beta_{0} b_{0}^{*} b_{0}-\beta \sum_{j=1}^{N} b_{j}^{*} b_{j}}\left(b_{0}^{*} b_{0}-\sum_{k=0}^{N}\left|\left(U_{1} \ldots U_{N} e\right)_{k}\right|^{2} b_{k}^{*} b_{k}\right)\right]
\end{aligned}
$$

Finally, by Lemma 2.2, (2.16), (2.17), and by (3.3), we obtain

$$
\begin{aligned}
& \operatorname{Ent}(\rho(N \tau) \mid \rho)= \\
& \left.\frac{\beta-\beta_{0}}{Z\left(\beta, \beta_{0}-\beta\right)} \operatorname{Tr}_{\mathscr{H}}\left[e^{-\beta_{0} b_{0}^{*} b_{0}-\beta \sum_{j=1}^{N} b_{j}^{*} b_{j}}\left(\left(1-|z|^{2 N}\right) b_{0}^{*} b_{0}-\sum_{k=1}^{N}|w|^{2}|z|^{2 N-2 k} b_{k}^{*} b_{k}\right)\right)\right] \\
& =\frac{\left(\beta_{0}-\beta\right)\left(e^{\beta_{0}}-e^{\beta}\right)}{\left(e^{\beta_{0}}-1\right)\left(e^{\beta}-1\right)}\left(1-|z|^{2 N}\right),
\end{aligned}
$$

that proves (4.8). 
Remark 4.4 The relative entropy defined by (4.7) is non-negative. In contrast to invariant total entropy (Lemma 4.2), the relative entropy (4.8) is increasing monotonously with $N \rightarrow \infty$ for $|z|<1$ (see Lemma 1.1, Remark 1.2). It converges to the limit:

$$
\lim _{N \rightarrow \infty} \operatorname{Ent}(\rho(N \tau) \mid \rho)=\left(\beta-\beta_{0}\right)\left[\frac{1}{e^{\beta_{0}}-1}-\frac{1}{e^{\beta}-1}\right] \geq 0
$$

which is positive for $\beta_{0} \neq \beta$. The limit (4.12) gives asymptotic amount of the entropy production, when one starts with the initial state corresponding to (4.1) and then consider $N \tau \rightarrow \infty$, see [BJM].

\section{Evolution of Subsystems}

\subsection{Convergence to Equilibrium}

Subsystem $\mathcal{S}$. We start with the simplest subsystem $\mathcal{S}$. Let the initial state of the total system $\mathcal{S}+\mathcal{C}$ in (1.1) be a tensor-product of the corresponding density matrices $\rho=\rho_{S} \otimes \rho_{C}$, see $(\mathrm{H} 1)$. Then for $t \geq 0$ the state $\omega_{\mathcal{S}}^{t}(\cdot)$ of the subsystem $\mathcal{S}$ is given on the Weyl $C^{*}$-algebra $\mathscr{A}\left(\mathscr{H}_{0}\right)$ by

$$
\omega_{\mathcal{S}}^{t}(\cdot):=\omega_{\rho(t)}(\cdot \otimes \mathbb{1}) .
$$

For $\zeta=(\alpha, 0, \ldots, 0) \in \mathbb{C}^{N+1}$, let us consider the Weyl operator $W(\zeta)=\widehat{w}(\alpha) \otimes \mathbb{1} \otimes \ldots \otimes \mathbb{1}$ (2.3). By virtue of (2.8), (2.21) and (5.1), we obtain for $t=m \tau(1 \leq m \leq N)$ :

$$
\omega_{\mathcal{S}}^{m \tau}(\widehat{w}(\alpha))=\omega_{\rho(m \tau)}(W(\zeta))=\omega_{\rho}\left(W\left(U_{1} \ldots U_{m} \zeta\right)\right)
$$

Then for components $\left\{\left(U_{1} \ldots U_{m} \zeta\right)_{k}\right\}_{k=0}^{N}$ of the vector $U_{1} \ldots U_{m} \zeta$ in (5.2), one obtains the expression:

$$
\left(U_{1} \ldots U_{m} \zeta\right)_{k}= \begin{cases}e^{i m \tau \epsilon}(g z)^{m} \alpha & (k=0) \\ e^{i m \tau \epsilon} g w(g z)^{m-k} \alpha & (1 \leqslant k<m) \\ e^{i m \tau \epsilon} g w \alpha & (k=m) \\ 0 & (m<k \leqslant N)\end{cases}
$$

which follows from Remark 2.3.

If the initial density matrices: $\rho=\rho_{S} \otimes \rho_{C}$ corresponds to the product of Gibbs quasi-free states for different temperatures as in (4.1), then (5.2) and Lemma4.1 yield

$$
\omega_{\mathcal{S}}^{m \tau}(\widehat{w}(\alpha))=\exp \left[-\frac{|\alpha|^{2}}{4} \frac{1+e^{-\beta}}{1-e^{-\beta}}-\frac{\left|z^{m} \alpha\right|^{2}}{4}\left(\frac{1+e^{-\beta_{0}}}{1-e^{-\beta_{0}}}-\frac{1+e^{-\beta}}{1-e^{-\beta}}\right)\right]
$$

Note that for any moment $t=m \tau$ the state $\omega_{\mathcal{S}}^{m \tau}(\cdot)$ is a quasi-free Gibbs equilibrium state with parameter $\beta^{*}(m \tau)$ which satisfies the equation

$$
\frac{1+e^{-\beta^{*}(m \tau)}}{1-e^{-\beta^{*}(m \tau)}}=|z|^{2 m} \frac{1+e^{-\beta_{0}}}{1-e^{-\beta_{0}}}+\left(1-|z|^{2 m}\right) \frac{1+e^{-\beta}}{1-e^{-\beta}} .
$$


This equation yields that either $\beta \leq \beta^{*}(m \tau) \leq \beta_{0}$, or $\beta_{0} \leq \beta^{*}(m \tau) \leq \beta$.

For $m \rightarrow \infty(N \rightarrow \infty)$ the Weyl characteristic function (5.4) has the limit

$$
\lim _{m \rightarrow \infty} \omega_{\mathcal{S}}^{m \tau}(\widehat{w}(\alpha))=\exp \left[-\frac{|\alpha|^{2}}{4} \frac{1+e^{-\beta}}{1-e^{-\beta}}\right]
$$

Hence, in the limit $t \rightarrow \infty$ the subsystem $\mathcal{S}$ evolves from the Gibbs equilibrium state with parameter $\beta_{0}$ to another equilibrium state with parameter $\beta$ imposed by the chain $\mathcal{C}$.

Subsystem $\mathcal{S}_{1}$. The initial state $\omega_{\mathcal{S}_{1}}^{0}(\cdot)=\left.\omega_{\mathcal{S}_{1}}^{t}(\cdot)\right|_{t=0}$ of this subsystem corresponds to a onepoint reduced density matrix or to the partial trace on the CCR Weyl algebra $\mathscr{A}\left(\mathscr{H}_{1}\right)$ :

$$
\omega_{\mathcal{S}_{1}}^{0}(\widehat{w}(\alpha))=\omega_{\rho}\left(\mathbb{1} \otimes \widehat{w}(\alpha) \otimes \bigotimes_{k=2}^{N} \mathbb{1}\right)=\exp \left[-\frac{|\alpha|^{2}}{4} \frac{1+e^{-\beta}}{1-e^{-\beta}}\right] .
$$

Now we choose vector $\left(\zeta^{1}\right)^{\operatorname{tr}}:=(0, \alpha, 0, \ldots, 0) \in \mathbb{C}^{N+1}$. Then

$$
\omega_{\mathcal{S}_{1}}^{m \tau}(\widehat{w}(\alpha))=\omega_{\rho(m \tau)}\left(W\left(\zeta^{(1)}\right)\right)=\omega_{\rho_{S} \otimes \rho_{C}}\left(W\left(U_{1} \ldots U_{m} \zeta^{(1)}\right)\right)
$$

for $1<m \leq N$. By Remark 2.3, the components $\left\{\left(U_{1} \ldots U_{m} \zeta^{(1)}\right)_{k}\right\}_{k=0}^{N}$ are:

$$
\left(U_{1} \ldots U_{m} \zeta\right)_{k}= \begin{cases}e^{i m \tau \epsilon} g w \alpha & (k=0) \\ e^{i m \tau \epsilon} \delta_{k, 1} g \bar{z} \alpha & (1 \leqslant k<m) \\ 0 & (m \leqslant k \leqslant N) .\end{cases}
$$

Then, we have

$$
\omega_{\mathcal{S}_{1}}^{m \tau}(\widehat{w}(\alpha))=\exp \left[-\frac{|\alpha|^{2}}{4} \frac{1+e^{-\beta}}{1-e^{-\beta}}-\frac{|w \alpha|^{2}}{4}\left(\frac{1+e^{-\beta_{0}}}{1-e^{-\beta_{0}}}-\frac{1+e^{-\beta}}{1-e^{-\beta}}\right)\right]
$$

for any $1<m \leq N$. Therefore, the initial state (5.7) changes to (5.10) after the first act of interaction on the interval $[0, \tau)$ and there is no further evolution of this state for $t>\tau$.

Note that (5.10) is characteristic function of a quasi-free Gibbs equilibrium state with parameter $\beta^{*}$, which satisfies the equation

$$
\frac{1+e^{-\beta^{*}}}{1-e^{-\beta^{*}}}=|w|^{2} \frac{1+e^{-\beta_{0}}}{1-e^{-\beta_{0}}}+\left(1-|w|^{2}\right) \frac{1+e^{-\beta}}{1-e^{-\beta}} .
$$

Again, this equation implies that either $\beta \leq \beta^{*} \leq \beta_{0}$, or $\beta_{0} \leq \beta^{*} \leq \beta$.

Evolution of $\mathcal{S}_{1}$ has a transparent interpretation: after the one act of interaction during the time $t \in[0, \tau)$, subsystem $\mathcal{S}_{1}$ relaxes to an intermediate equilibrium with the subsystem $\mathcal{S}$. This results in a shift of initial parameter $\beta$ to $\beta^{*}$, which rests unchangeable since there is no perturbations of subsystem $\mathcal{S}_{1}$ for $t>\tau$.

Subsystem $\mathcal{S}_{m}$. For $1<m \leq N$ the initial state $\omega_{\mathcal{S}_{m}}^{0}(\cdot)=\left.\omega_{\mathcal{S}_{m}}^{t}(\cdot)\right|_{t=0}$ of this subsystem is defined on the CCR Weyl algebra $\mathscr{A}\left(\mathscr{H}_{m}\right)$ by the partial trace :

$$
\omega_{\mathcal{S}_{m}}^{0}(\widehat{w}(\alpha))=\omega_{\rho}\left(\bigotimes_{k=0}^{m-1} \mathbb{1} \otimes \widehat{w}(\alpha) \otimes \bigotimes_{k=m+1}^{N} \mathbb{1}\right)=\exp \left[-\frac{|\alpha|^{2}}{4} \frac{1+e^{-\beta}}{1-e^{-\beta}}\right] .
$$


Now we choose vector $\left(\zeta^{m}\right)^{\operatorname{tr}}:=(0, \ldots, 0, \alpha, 0, \ldots, 0) \in \mathbb{C}^{N+1}$, where $\alpha$ occupies the $m+1$ position. Consequently

$$
\omega_{\mathcal{S}_{m}}^{m \tau}(\widehat{w}(\alpha))=\omega_{\rho(m \tau)}\left(W\left(\zeta^{(m)}\right)\right)=\omega_{\rho_{S} \otimes \rho_{C}}\left(W\left(U_{1} \ldots U_{m} \zeta^{(m)}\right)\right) .
$$

The components $\left\{\left(U_{1} \ldots U_{m} \zeta^{(m)}\right)_{k}\right\}_{k=0}^{N}$ are:

$$
\left(U_{1} \ldots U_{m} \zeta^{(m)}\right)_{k}= \begin{cases}e^{i m \tau \epsilon} g w(g z)^{m-1} \alpha & (k=0) \\ e^{i m \tau \epsilon} g^{2} w^{2}(g z)^{m-k-1} \alpha & (1 \leqslant k<m) \\ e^{i m \tau \epsilon} g \bar{z} \alpha, & (k=m) \\ 0 & (m<k \leqslant N) .\end{cases}
$$

which again follows from Remark 2.3. Then evolution of the state of subsystem $\mathcal{S}_{m}$ is:

$$
\begin{aligned}
& \omega_{\mathcal{S}_{m}}^{m \tau}(\widehat{w}(\alpha))= \\
& \exp \left[-\frac{|\alpha|^{2}}{4} \frac{1+e^{-\beta}}{1-e^{-\beta}}-\frac{|w \alpha|^{2}}{4}|z|^{2(m-1)}\left(\frac{1+e^{-\beta_{0}}}{1-e^{-\beta_{0}}}-\frac{1+e^{-\beta}}{1-e^{-\beta}}\right)\right] .
\end{aligned}
$$

Note that interaction for $t \in[(m-1) \tau, m \tau)$ push out the subsystem $\mathcal{S}_{m}$ from the Gibbs equilibrium state (5.11), but its effect attenuates for large $m$ :

$$
\lim _{m \rightarrow \infty} \omega_{\mathcal{S}_{m}}^{m \tau}(\widehat{w}(\alpha))=\exp \left[-\frac{|\alpha|^{2}}{4} \frac{1+e^{-\beta}}{1-e^{-\beta}}\right] .
$$

Again, this is evolution of a quasi-free Gibbs equilibrium state with time-dependent inverse temperature parameter $\beta^{* *}(m \tau)$, which satisfies the equation

$$
\frac{1+e^{-\beta^{* *}(m \tau)}}{1-e^{-\beta^{* *}(m \tau)}}=|w|^{2}|z|^{2(m-1)} \frac{1+e^{-\beta_{0}}}{1-e^{-\beta_{0}}}+\left(1-|w|^{2}|z|^{2(m-1)}\right) \frac{1+e^{-\beta}}{1-e^{-\beta}} .
$$

As above, the value of the parameter $\beta^{* *}(m \tau)$ is always between $\beta_{0}$ and $\beta$.

To interpret the evolution of $\mathcal{S}_{m}$ and the coincidence between (5.15) and (5.6) note that the state of the subsystem $\mathcal{S}$ relaxes to that of initial state of the chain $\mathcal{C}$, see (5.6). Therefore, after interaction of the subsystem $\mathcal{S}_{m}$, i.e. at the moment $t=m \tau$, its parameter $\beta^{* *}(m \tau)$ has a value between $\beta$ and $\beta^{*}((m-1) \tau)$ since (5.5) and (5.16) yield

$$
\frac{1+e^{-\beta^{* *}(m \tau)}}{1-e^{-\beta^{* *}(m \tau)}}=|w|^{2} \frac{1+e^{-\beta^{*}((m-1) \tau)}}{1-e^{-\beta^{*}((m-1) \tau)}}+\left(1-|w|^{2}\right) \frac{1+e^{-\beta}}{1-e^{-\beta}} .
$$

As in the case $m=1$, there is no further evolution: $\omega_{\mathcal{S}_{m}}^{n \tau}=\omega_{\mathcal{S}_{m}}^{m \tau}$ for $n \geqslant m$.

Next, we consider the composed subsystems $\mathcal{S}+\mathcal{S}_{m}$ and $\mathcal{S}_{m-n}+\mathcal{S}_{m}$. Our aim is to study the eventual correlations imposed by repeated perturbations due to $\mathcal{S}$.

Subsystem $\mathcal{S}+\mathcal{S}_{m}$. For $1<m \leqslant N$ the initial state $\omega_{\mathcal{S}+\mathcal{S}_{m}}^{0}(\cdot)=\left.\omega_{\mathcal{S}+\mathcal{S}_{m}}^{t}(\cdot)\right|_{t=0}$ of this composed subsystem is defined by the partial trace on the Weyl $C^{*}$-algebra $\mathscr{A}\left(\mathscr{H}_{0} \otimes \mathscr{H}_{m}\right) \approx$ $\mathscr{A}\left(\mathscr{H}_{0}\right) \otimes \mathscr{A}\left(\mathscr{H}_{m}\right)$ by:

$$
\begin{aligned}
& \omega_{\mathcal{S}+\mathcal{S}_{m}}^{0}\left(\widehat{w}\left(\alpha_{0}\right) \otimes \widehat{w}\left(\alpha_{1}\right)\right):=\omega_{\rho}\left(\widehat{w}\left(\alpha_{0}\right) \otimes \bigotimes_{k=1}^{m-1} \mathbb{1} \otimes \widehat{w}\left(\alpha_{1}\right) \otimes \bigotimes_{k=m+1}^{N} \mathbb{1}\right) \\
& =\exp \left[-\frac{\left|\alpha_{0}\right|^{2}}{4} \frac{1+e^{-\beta_{0}}}{1-e^{-\beta_{0}}}\right] \exp \left[-\frac{\left|\alpha_{1}\right|^{2}}{4} \frac{1+e^{-\beta}}{1-e^{-\beta}}\right] .
\end{aligned}
$$


This is the characteristic function of the product state corresponding to two isolated systems with different temperatures. If one defines vector $\left(\zeta^{(0, m)}\right)^{\operatorname{tr}}:=\left(\alpha_{0}, 0, \ldots, 0, \alpha_{1}, 0, \ldots, 0\right) \in$ $\mathbb{C}^{N+1}$, where $\alpha_{1}$ occupies the $m+1$ position, then

$$
\omega_{\mathcal{S}+\mathcal{S}_{m}}^{m \tau}\left(\widehat{w}\left(\alpha_{0}\right) \otimes \widehat{w}\left(\alpha_{1}\right)\right)=\omega_{\rho(m \tau)}\left(W\left(\zeta^{(0, m)}\right)\right)=\omega_{\rho_{S} \otimes \rho_{C}}\left(W\left(U_{1} \ldots U_{m} \zeta^{(0, m)}\right)\right) .
$$

The components $\left\{\left(U_{1} \ldots U_{m} \zeta^{(0, m)}\right)_{k}\right\}_{k=0}^{N}$ are deduced from Remark 2.3:

$$
\left(U_{1} \ldots U_{m} \zeta^{(0, m)}\right)_{k}= \begin{cases}e^{i m \tau \epsilon}(g z)^{m-1}\left[g z \alpha_{0}+g w \alpha_{1}\right], & (k=0) \\ e^{i m \tau \epsilon}(g z)^{m-k-1} g^{2}\left[w z \alpha_{0}+w^{2} \alpha_{1}\right], & (1 \leqslant k<m) \\ e^{i m \tau \epsilon}\left[g w \alpha_{0}+g \bar{z} \alpha_{1}\right], & (k=m) \\ 0 & (m<k \leqslant N) .\end{cases}
$$

Together with (2.8), one gets for $m \rightarrow \infty$ :

$$
\begin{aligned}
& \omega_{\mathcal{S}+\mathcal{S}_{m}}^{m \tau}\left(\widehat{w}\left(\alpha_{0}\right) \otimes \widehat{w}\left(\alpha_{1}\right)\right) \\
& =\exp \left[-\frac{1}{4}\left|z \alpha_{0}+w \alpha_{1}\right|^{2}|z|^{2(m-1)} \frac{1+e^{-\beta_{0}}}{1-e^{-\beta_{0}}}\right] \\
& \times \exp \left[-\frac{1}{4}\left|z \alpha_{0}+w \alpha_{1}\right|^{2}\left(1-|z|^{2(m-1)}\right) \frac{1+e^{-\beta}}{1-e^{-\beta}}\right] \exp \left[-\frac{1}{4}\left|w \alpha_{0}+\bar{z} \alpha_{1}\right|^{2} \frac{1+e^{-\beta}}{1-e^{-\beta}}\right] \\
& \longrightarrow \exp \left[-\frac{1}{4}\left(\left|\alpha_{0}\right|^{2}+\left|\alpha_{1}\right|^{2}\right) \frac{1+e^{-\beta}}{1-e^{-\beta}}\right] .
\end{aligned}
$$

Hence, in this limit the composed subsystem $\mathcal{S}+\mathcal{S}_{m}$ evolves from the product of two quasi-free equilibrium states (5.17) with different parameters $\beta_{0}$ and $\beta$ to the product of quasi-free equilibrium states for the same parameter $\beta$ imposed by repeated interaction with the chain $\mathcal{C}$, when $m \rightarrow \infty$. Interpretation is similar to the case Subsystem $\mathcal{S}_{m}$.

Subsystem $\mathcal{S}_{m-n}+\mathcal{S}_{m}$. We suppose that $1<(m-n)<m \leqslant N$. Then the initial state $\left.\omega_{\mathcal{S}_{m-n}+\mathcal{S}_{m}}^{t}(\cdot)\right|_{t=0}$ of this composed subsystem is the partial trace over the Weyl $C^{*}$-algebra $\mathscr{A}\left(\mathscr{H}_{m-n} \otimes \mathscr{H}_{m}\right) \approx \mathscr{A}\left(\mathscr{H}_{m-n}\right) \otimes \mathscr{A}\left(\mathscr{H}_{m}\right):$

$$
\begin{aligned}
& \omega_{\mathcal{S}_{m-n}+\mathcal{S}_{m}}^{0}\left(\widehat{w}\left(\alpha_{1}\right) \otimes \widehat{w}\left(\alpha_{2}\right)\right):= \\
& \omega_{\rho}\left(\bigotimes_{k=0}^{m-n-1} \mathbb{1} \otimes \widehat{w}\left(\alpha_{1}\right) \otimes \bigotimes_{k=m-n+1}^{m-1} \mathbb{1} \otimes \widehat{w}\left(\alpha_{2}\right) \otimes \bigotimes_{k=m+1}^{N} \mathbb{1}\right)= \\
& =\exp \left[-\frac{\left|\alpha_{1}\right|^{2}}{4} \frac{1+e^{-\beta}}{1-e^{-\beta}}\right] \exp \left[-\frac{\left|\alpha_{2}\right|^{2}}{4} \frac{1+e^{-\beta}}{1-e^{-\beta}}\right] .
\end{aligned}
$$

This is the characteristic function of the product state corresponding to two isolated systems with the same temperatures.

We define vector $\left(\zeta^{(m-n, m)}\right)^{\operatorname{tr}}:=\left(0,0, \ldots, 0, \alpha_{1}, 0, \ldots, 0, \alpha_{2}, 0, \ldots, 0\right) \in \mathbb{C}^{N+1}$, where $\alpha_{1}$ occupies the $m-n+1$ position, and $\alpha_{2}$ occupies the $m+1$ position, then

$$
\begin{aligned}
& \omega_{\mathcal{S}_{m-n}+\mathcal{S}_{m}}^{m \tau}\left(\widehat{w}\left(\alpha_{1}\right) \otimes \widehat{w}\left(\alpha_{2}\right)\right)= \\
& \omega_{\rho(m \tau)}\left(W\left(\zeta^{(m-n, m)}\right)\right)=\omega_{\rho_{S} \otimes \rho_{C}}\left(W\left(U_{1} \ldots U_{m} \zeta^{(m-n, m)}\right)\right) .
\end{aligned}
$$


With help of Remark 2.3 we can calculate the values of components $\left\{\left(U_{1} \ldots U_{m} \zeta^{(m-n, m)}\right)_{k}\right\}_{k=0}^{N}$ :

$$
\begin{gathered}
\quad\left(U_{1} \ldots U_{m} \zeta^{(m-n, m)}\right)_{k}= \\
=\left\{\begin{array}{ll}
e^{i m \tau \epsilon}(g z)^{m-n-1} g w\left[\alpha_{1}+(g z)^{n} \alpha_{2}\right] & (k=0) \\
e^{i m \tau \epsilon}\left[g^{2} w^{2}(g z)^{m-n-k-1} \alpha_{1}+g^{2} w^{2}(g z)^{m-k-1} \alpha_{2}\right] & (1 \leqslant k<m-n) \\
e^{i m \tau \epsilon}\left[g \bar{z} \alpha_{1}+g^{2} w^{2}(g z)^{m-k-1} \alpha_{2}\right] & (k=m-n) \\
e^{i m \tau \epsilon} g^{2} w^{2}(g z)^{m-k-1} \alpha_{2} & (m-n<k<m) \\
e^{i m \tau \epsilon} g \bar{z} \alpha_{2} & (k=m) \\
0 & (m<k \leqslant N)
\end{array} .\right.
\end{gathered}
$$

When $m \rightarrow \infty$, then for any fixed $n$ we obtain for (5.22):

$$
\begin{aligned}
& \omega_{\mathcal{S}_{m-n}+\mathcal{S}_{m}}^{m \tau}\left(\widehat{w}\left(\alpha_{1}\right) \otimes \widehat{w}\left(\alpha_{2}\right)\right) \\
& =\exp \left[-\frac{1}{4}|w|^{2}\left|\alpha_{1}+(g z)^{n+1} \alpha_{2}\right|^{2}|z|^{2(m-n-1)} \frac{1+e^{-\beta_{0}}}{1-e^{-\beta_{0}}}\right] \\
& \times \exp \left[-\frac{1}{4}\left\{|w|^{2}\left(1-|z|^{2(m-n-1)}\right)+|z|^{2}\right\}\left|\alpha_{1}\right|^{2} \frac{1+e^{-\beta}}{1-e^{-\beta}}\right] \\
& \times \exp \left[-\frac{1}{4}\left(1-|w|^{2}|z|^{2(m-1)}\right)\left|\alpha_{2}\right|^{2} \frac{1+e^{-\beta}}{1-e^{-\beta}}\right] . \\
& \longrightarrow \exp \left[-\frac{1}{4}\left(\left|\alpha_{1}\right|^{2}+\left|\alpha_{2}\right|^{2}\right) \frac{1+e^{-\beta}}{1-e^{-\beta}}\right] .
\end{aligned}
$$

Therefore, in this limit, the composed subsystem $\mathcal{S}_{m-n}+\mathcal{S}_{m}$ evolves from the initial product of two quasi-free equilibrium states (5.21) to the same final state, although for a finite $m$ the evolution (5.24) is nontrivial. This again easily understandable taking into account our analysis of Subsystem $\mathcal{S}_{m}$ and Subsystem $\mathcal{S}+\mathcal{S}_{m}$.

Consider now the case of a fixed $s:=m-n \geqslant 1$. Then the limit in (5.24) is

$$
\begin{aligned}
& \lim _{m \rightarrow \infty} \omega_{\mathcal{S}_{s}+\mathcal{S}_{m}}^{m \tau}\left(\widehat{w}\left(\alpha_{1}\right) \otimes \widehat{w}\left(\alpha_{2}\right)\right)= \\
& =\exp \left[-\frac{1}{4}|w|^{2}|z|^{2(s-1)}\left|\alpha_{1}\right|^{2}\left\{\frac{1+e^{-\beta_{0}}}{1-e^{-\beta_{0}}}-\frac{1+e^{-\beta}}{1-e^{-\beta}}\right\}\right] \times \\
& \times \exp \left[-\frac{1}{4}\left|\alpha_{1}\right|^{2} \frac{1+e^{-\beta}}{1-e^{-\beta}}\right] \exp \left[-\frac{1}{4}\left|\alpha_{2}\right|^{2} \frac{1+e^{-\beta}}{1-e^{-\beta}}\right] \\
& =\exp \left[-\frac{1}{4}\left|\alpha_{1}\right|^{2} \frac{1+e^{-\beta^{* *}(s \tau)}}{1-e^{-\beta^{* *}(s \tau)}}\right] \exp \left[-\frac{1}{4}\left|\alpha_{2}\right|^{2} \frac{1+e^{-\beta}}{1-e^{-\beta}}\right],
\end{aligned}
$$

where $\beta^{* *}(s \tau)$ verifies equation (5.16). Hence, in this case the limit state (5.25) is the product of quasi-free Gibbs states with different parameters $\beta^{* *}(s \tau)$ and $\beta$. This means that subsystem $\mathcal{S}_{s}$ keeps a memory about perturbation at the moment $t=s \tau$, when the parameter $\beta^{*}(s \tau)(5.5)$ of subsystem $\mathcal{S}$ was still different from $\beta$.

Note that (5.25) coincides with the product state (5.21) when $s \rightarrow \infty$.

Subsystem $\mathcal{S}_{\sim n}$. To define $\mathcal{S}_{\sim n}$ for $0 \leqslant n \leqslant k \leqslant N$, we divide the total system at the moment $t=k \tau$ into two subsystems: $\mathcal{S}_{n, k}+\mathcal{C}_{n, k}$. Here

$$
\mathcal{S}_{n, k}:=\mathcal{S}+\mathcal{S}_{k}+\mathcal{S}_{k-1}+\cdots+\mathcal{S}_{k-n+1},\left(\mathcal{S}_{0, k}:=\mathcal{S}\right)
$$


whereas (see definitions in Section 1.1)

$$
\mathcal{C}_{n, k}:=\mathcal{S}_{N}+\cdots+\mathcal{S}_{k+1}+\mathcal{S}_{k-n}+\cdots+\mathcal{S}_{1}
$$

We mean that $\mathcal{S}_{\sim n}$ is an entire "object" whose entity is $\mathcal{S}_{n, k}$ at the moment $t=k \tau$ ( $k=n, n+1, \cdots, N)$. As time is running, the elementary subsystems $\mathcal{S}_{k}$ in $\mathcal{S}_{\sim n}$ are replacing. We study the behaviour of $\mathcal{S}_{\sim n}$ for large $t=k \tau$, i.e., the $k$-dependence of the "state" of $\mathcal{S}_{n, k}$ at $t=k \tau$.

For any fixed $t=k \tau$ we can decompose the Hilbert space $\mathscr{H}$ into tensor product $\mathscr{H}=\mathscr{H}_{s} \otimes \mathscr{H}_{c}$. Here $\mathscr{H}_{s}$ is the Hilbert space of subsystem (5.26) and $\mathscr{H}_{c}$ corresponds to subsystem (5.27):

$$
\mathscr{H}_{s}:=\mathscr{H}_{0} \otimes \bigotimes_{j=1}^{n} \mathscr{H}_{k-j+1}, \quad \mathscr{H}_{c}:=\bigotimes_{j=1}^{k-n} \mathscr{H}_{j} \otimes \bigotimes_{j=k+1}^{N} \mathscr{H}_{j}
$$

For a density matrix $\varrho$ on $\mathscr{H}$, we introduce the reduced density matrix $\varrho_{s}$ on $\mathscr{H}_{s}$ as the partial trace over $\mathscr{H}_{c}$ :

$$
\varrho_{s}:=\operatorname{Tr}_{\mathscr{H}_{c}} \varrho .
$$

To avoid a possible confusion causing by the fact that all $\mathscr{H}_{j}, j=0,1, \ldots$ are identical to $\mathscr{F}$ and by the change of components with time, we treat the Weyl algebra on the subsystem and the corresponding reduced density matrix of $\rho \in \mathfrak{C}_{1}(\mathscr{H})$ in the following way. For $n \leqslant N$ on the Fock space $\mathcal{F}^{\otimes(n+1)}$ we consider the Weyl operator

$$
W_{n}(\zeta)=\exp \left[i \frac{\langle\zeta, \tilde{b},\rangle+\langle\tilde{b}, \zeta\rangle}{\sqrt{2}}\right]
$$

where $\zeta \in \mathbb{C}^{n+1}, \tilde{b}_{0}, \cdots, \tilde{b}_{n}$ and $\tilde{b}_{0}^{*}, \cdots, \tilde{b}_{n}^{*}$ are the annihilation and the creation operators in $\mathcal{F}^{\otimes(n+1)}$ satisfying the corresponding CCR, and

$$
\langle\zeta, \tilde{b}\rangle=\sum_{j=0}^{n} \bar{\zeta}_{j} \tilde{b}_{j}, \quad\langle\tilde{b}, \zeta\rangle=\sum_{j=0}^{n} \zeta_{j} \tilde{b}_{j}^{*}
$$

By $\mathscr{A}\left(\mathscr{F}^{\otimes(n+1)}\right)$, we denote the $C^{*}$ algebra generated by the Weyl operators (5.30). For any subset $J \subset\{1,2, \cdots, N\}$, we define the operation of taking the partial trace

$$
R^{J}: \mathfrak{C}_{1}\left(\mathscr{F}^{\otimes(N+1)}\right) \ni \rho \longmapsto R^{J} \rho \in \mathfrak{C}_{1}\left(\mathscr{F}^{\otimes(N+1-|J|)}\right)
$$

by

$$
\omega_{R^{J} \rho}\left(W_{N-|J|}(\zeta)\right)=\omega_{\rho}\left(W_{N}\left(r_{J} \zeta\right)\right)
$$

Here the mapping

$$
r_{J}: \mathbb{C}^{N+1-|J|} \ni \zeta \longmapsto r_{J} \zeta \in \mathbb{C}^{N+1}
$$

is defined by

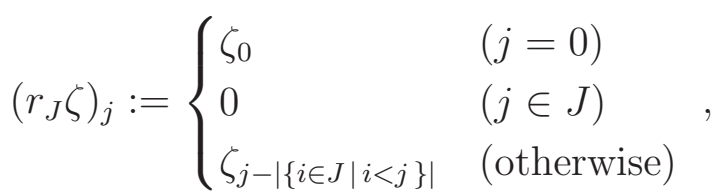


where $|A|$ denotes the cardinality of the set $A$.

Since all $\mathscr{H}_{1}, \mathscr{H}_{2}, \cdots$ are identical to $\mathscr{F}$, we do not care to distinguish the spaces

$$
\bigotimes_{j \in\{0,1, \cdots, N\} \backslash J} \mathscr{H}_{j} \text { and } \bigotimes_{j \in\{0,1, \cdots, N\} \backslash J^{\prime}} \mathscr{H}_{j}
$$

when $J \neq J^{\prime}$, but $|J|=\left|J^{\prime}\right|$, and consider them as the same space $\mathscr{F}^{\otimes(N+1-|J|)}$. Instead, we pay attention to distinguishing projections

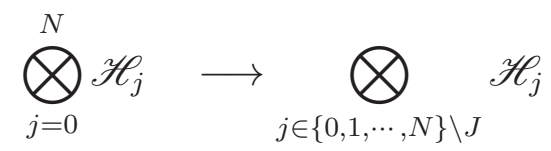

for different subsets $J \subset\{1,2, \cdots, N\}$ with same $|J|$.

Since we treat $\mathcal{S}_{n, k}$ at time $t=k \tau$ for $k=n, n+1, \cdots$ as the result of the time evolution of a single subsystem $\mathcal{S}_{\sim n}$, we define its state at the moment $t=k \tau$ by the reduced density matrix $\left\{\rho_{s}(k \tau)\right\}_{k \geqslant n}$ of this subsystem as follows:

$$
\rho_{s}(k \tau):=R^{\{1, \cdots, k-n, k+1, \cdots, N\}}(\rho(k \tau))=R^{\{1, \cdots, k-n, k+1, \cdots, N\}} T_{k \tau}(\rho),
$$

see (2.12). Taking into account Lemma 4.2 and identity $\left\langle r_{J} \zeta, r_{J} \zeta\right\rangle_{\mathbb{C}^{N+1}}=\langle\zeta, \zeta\rangle_{\mathbb{C}^{N+1-|J|}}$, one readily obtains the following result.

Lemma 5.1 For the initial density matrix (4.1),

$$
\begin{gathered}
\omega_{\rho_{s}(k \tau)}\left(W_{n}(\zeta)\right)=\omega_{R^{J_{n, k}} \rho(k \tau)}\left(W_{n}(\zeta)\right) \\
=\exp \left[-\frac{\left|\left(U_{1} \ldots U_{k} r_{J_{n, k}} \zeta\right)_{0}\right|^{2}}{4}\left(\frac{1+e^{-\beta_{0}}}{1-e^{-\beta_{0}}}-\frac{1+e^{-\beta}}{1-e^{-\beta}}\right)-\frac{\langle\zeta, \zeta\rangle}{4} \frac{1+e^{-\beta}}{1-e^{-\beta}}\right]
\end{gathered}
$$

holds, where $J_{n, k}=\{1,2, \cdots, k-n, k+1, \cdots, N\}$.

To study the limit $k \rightarrow \infty$ and $N \rightarrow \infty(k \leqslant N)$ for a fixed $n$, we note that $\left(U_{1} \ldots U_{k} r_{J_{n, k}} \zeta\right)_{0} \rightarrow 0$ follows from $(2.16)$ and $|z|<1$. Lemma 5.1 implies that

$$
\lim _{k \rightarrow \infty} \omega_{\rho_{s}(k \tau)}\left(W_{n}(\zeta)\right)=\exp \left[-\frac{\langle\zeta, \zeta\rangle}{4} \frac{1+e^{-\beta}}{1-e^{-\beta}}\right]=\omega_{\rho_{n}^{(\beta)}}\left(W_{n}(\zeta)\right)
$$

where by the Araki-Segal theorem and irreducibility of the CCR algebra $\mathscr{A}(\mathscr{F} \otimes(n+1))$ one has

$$
\rho_{n}^{(\beta)}=\exp \left[-\beta \sum_{j=0}^{n} \tilde{b}_{j}^{*} \tilde{b}_{j}\right] / Z(\beta)^{n+1}, Z(\beta)=\left(1-e^{-\beta}\right)^{-1} .
$$

Therefore, we proved the following statement:

Theorem 5.2 Let the initial state of the total system $\mathcal{S}+\mathcal{C}$ is defined by the density matrix (4.2): $\rho=\rho\left(\beta, \beta_{0}-\beta ; e\right)$. Then for any fixed $n$, the state $\omega_{\rho_{s}(k \tau)}(\cdot)$ of subsystem $\mathcal{S}_{n, k}$ converges to the equilibrium Gibbs state $\omega_{\rho_{n}^{(\beta)}}(\cdot)$ as $k \rightarrow \infty$ in the weak ${ }^{*}$-topology for the states on $\mathscr{A}(\mathscr{F} \otimes(n+1))$. 
Theorem 5.3 Under the same conditions as in Theorem 5.2, we obtain

$$
\lim _{k \rightarrow \infty} S\left(\rho_{s}(k \tau)\right)=S\left(\rho_{n}^{(\beta)}\right)
$$

Proof: Let the vector $\xi_{n, k} \in \mathbb{C}^{n+1}$ be defined by $\left(U_{1} \ldots U_{k} r_{J_{n, k}} \zeta\right)_{0}=:\left\langle\xi_{n, k}, \zeta\right\rangle$. Then $\left\langle\xi_{n, k}, \xi_{n, k}\right\rangle \rightarrow 0$ as $k \rightarrow \infty$ for a fixed $n$ and by Lemma 3.3, Lemma 5.1, we obtain that

$$
\begin{gathered}
S\left(\rho_{s}(k \tau)\right)=n \sigma\left(\frac{1+e^{-\beta}}{1-e^{-\beta}}\right)+\sigma\left(\frac{1+e^{-\beta}}{1-e^{-\beta}}+\left\langle\xi_{n, k}, \xi_{n, k}\right\rangle\left(\frac{1+e^{-\beta_{0}}}{1-e^{-\beta_{0}}}-\frac{1+e^{-\beta}}{1-e^{-\beta}}\right)\right) \\
\longrightarrow(n+1) \sigma\left(\frac{1+e^{-\beta}}{1-e^{-\beta}}\right)=S\left(\rho_{n}^{(\beta)}\right) .
\end{gathered}
$$

Remark 5.4 The local entropy decreases or increases according to $\beta>\beta_{0}$ or $\beta<\beta_{0}$, respectively.

\subsection{A Short-Time Limit for Repeated Perturbations}

The results in the Subsection 5.1 are essentially due explicit knowledge of the initial density matrix $(4.1)$ of the total system $\mathcal{S}+\mathcal{C}$. In this subsection, we show that the lack of this information is not decisive for certain results concerning the convergence to equilibrium if one considers the repeated interactions in a short-time limit.

We study this limit for the subsystem $\mathcal{S}$. We keep to consider the initial state of the system $\mathcal{S}+\mathcal{C}$ to be a product state with the density matrix

$$
\rho=\rho_{0} \otimes \bigotimes_{k=1}^{N} \rho_{k} \in \mathfrak{C}_{1}(\mathscr{H})
$$

see (2.7), but we essentially relax the conditions on $\rho_{0}$ and on $\left\{\rho_{k}\right\}_{k=1}^{N}$ :

(h2) $\operatorname{Tr}_{\mathscr{F}}\left(\rho_{1} a\right)=\operatorname{Tr}_{\mathscr{F}}\left(\rho_{1} a^{2}\right)=\operatorname{Tr}_{\mathscr{F}}\left(\rho_{1} a^{*}\right)=\operatorname{Tr}_{\mathscr{F}}\left(\rho_{1} a^{* 2}\right)=0$;

$$
\operatorname{Tr}_{\mathscr{F}}\left[\rho_{1}\left(a^{*} a\right)^{2}\right]<\infty \text {. }
$$

Remark 5.5 Note that hypothesis (h1)-(h3) are satisfied when the density matrices $\left\{\rho_{k}\right\}_{k=0}^{N}$ correspond to the gauge-invariant quasi-free states with parameter $\beta_{0}$ for $k=0$ and $\beta$ for $k=1,2, \ldots, N$, see (4.1). Then (h2) is due to the gauge invariance and one gets for (h3):

$$
\operatorname{Tr}_{\mathscr{F}}\left[\rho_{k}\left(a^{*} a\right)^{2}\right]=\left(2 n_{\beta}^{2}+n_{\beta}\right),
$$

where $n_{\beta}=\operatorname{Tr}_{\mathscr{F}}\left(\rho_{k} a^{*} a\right)=\left(e^{\beta}-1\right)^{-1}, k=1, \ldots, N$.

Below we denote by $\left|y a^{*}+\bar{y} a\right|$ the operator originated from the polar decomposition of the operator $y a^{*}+\bar{y} a=U\left|y a^{*}+\bar{y} a\right|$, where $U$ is the partial isometry on $\mathscr{F}$. 
Lemma 5.6 Under hypothesis (h1)-(h3), the following bounds hold:

$$
\begin{gathered}
\operatorname{Tr}_{\mathscr{F}}\left(\rho_{k} a^{*} a\right)<\infty, \\
\operatorname{Tr}_{\mathscr{F}}\left(\rho_{k}\left|y a^{*}+\bar{y} a\right|^{2}\right) \leqslant C|y|^{2}, \\
\operatorname{Tr}_{\mathscr{F}}\left(\rho_{k}\left|y a^{*}+\bar{y} a\right|^{3}\right) \leqslant C^{\prime}|y|^{3}, \\
\operatorname{Tr}_{\mathscr{F}}\left(\rho_{k}\left|y a^{*}+\bar{y} a\right|^{4}\right) \leqslant C^{\prime \prime}|y|^{4},
\end{gathered}
$$

for all $k=1, \ldots, N$. Here $C, C^{\prime}, C^{\prime \prime}$ are positive constants, which depend only on $\operatorname{Tr}\left[\rho_{1}\left(a^{*} a\right)^{2}\right]$.

Proof: The first bound (i) is a consequence of the Cauchy-Schwarz inequality and (h3). Applying the inequalities

$$
\begin{gathered}
\left|A+A^{*}\right|^{2} \leqslant\left|A+A^{*}\right|^{2}+\left|A-A^{*}\right|^{2}=2\left(A A^{*}+A^{*} A\right), \\
\left|A+A^{*}\right|^{4} \leqslant\left|A+A^{*}\right|^{4}+\left|A-A^{*}\right|^{4}+\left|A+i A^{*}\right|^{4}+\left|A-i A^{*}\right|^{4} \\
=4\left(A A^{*}+A^{*} A\right)^{2}+4\left(A^{2} A^{* 2}+A^{* 2} A^{2}\right),
\end{gathered}
$$

to $A=\bar{y} a$, we obtain (ii) and (iv). Finally, a combination of (ii), (iv) with the CauchySchwarz inequality yields (iii).

Theorem 5.7 Let $\tau \rightarrow 0, N \rightarrow \infty$ be short-time perturbation limit subject to demands: $\tau^{2} N \rightarrow \infty$ and $\tau^{3} N \rightarrow 0$. Then for any initial condition (5.34) verifying (h1)-(h3), the characteristic function $\omega_{\mathcal{S}}^{N \tau}(\widehat{w}(\theta))$ of the state for subsystem $\mathcal{S}$ at $t=N \tau$, converges to:

$$
\begin{aligned}
& \omega_{\mathcal{S}}(\widehat{w}(\theta)):=\lim _{\tau \rightarrow 0, N \rightarrow \infty} \omega_{\mathcal{S}}^{N \tau}(\widehat{w}(\theta))= \\
& \lim _{\tau \rightarrow 0, N \rightarrow \infty} \omega_{\rho(N \tau)}\left(W\left(\zeta_{\theta}\right)\right)=e^{-|\theta|^{2} \operatorname{Tr} \mathscr{F}\left[\rho_{1}\left(a^{*} a+a a^{*}\right)\right] / 4} .
\end{aligned}
$$

Here $\theta \in \mathbb{C}$ and the $(N+1)$-component vector is $\left(\zeta_{\theta}\right)^{\operatorname{tr}}:=(\theta, 0,0, \ldots, 0) \in \mathbb{C}^{N+1}$.

Remark 5.8 By (5.36) the state $\omega_{\mathcal{S}}^{N \tau}$ converges to $\omega_{\mathcal{S}}$ in the weak ${ }^{*}$-topology. From the right-hand side of (5.36) and Definition 3.1 we deduce that the limit state is gaugeinvariant and quasi-free with $h(\theta):=|\theta|^{2} \operatorname{Tr}_{\mathscr{F}}\left(\rho_{1} a^{*} a\right)$.

Remark 5.9 Recall that the state $\omega$ over the Weyl algebra $\mathscr{A}(\mathscr{F})=\overline{\mathscr{A}_{w}(\mathscr{F})}$ is regular, $C^{n}$-smooth or analytic, if the function (see (2.1))

$$
s \mapsto \omega(\widehat{w}(s \theta))=\omega\left(e^{i s \Phi(\theta) / \sqrt{2}}\right)
$$

is respectively continuous, $C^{n}$-smooth or analytic in the vicinity of $s=0$. In the last case the characteristic function $\omega(\widehat{w}(s \theta)$ ) (and therefore the state) is completely determined by

$$
\omega(\widehat{w}(s \theta))=\exp \left\{\sum_{m=1}^{\infty} \frac{i^{m} s^{m}}{m !} 2^{-m / 2} \omega^{T}\left(\Phi^{m}(\theta)\right)\right\} .
$$

Here $\left\{\omega^{T}\left(\Phi^{m}(\theta)\right)\right\}_{m=0}^{\infty}$ are truncated correlation functions defined recursively by relations

$$
\begin{aligned}
& \omega^{T}(\Phi(\theta)):=\omega(\Phi(\theta)), \\
& \omega^{T}\left(\Phi^{2}(\theta)\right):=\omega\left(\Phi^{2}(\theta)\right)-\omega(\Phi(\theta))^{2}, \\
& \omega^{T}\left(\Phi^{3}(\theta)\right):=\omega\left(\Phi^{3}(\theta)\right)-3 \omega\left(\Phi^{2}(\theta)\right) \omega(\Phi(\theta))+2 \omega(\Phi(\theta))^{3}, \text { etc }
\end{aligned}
$$

Lemma 5.6 implies that the states for density matrices $\rho_{1}=\rho_{2}=\ldots$ are $C^{4}$-smooth. 
Proof (of Theorem 5.7): By (h2) and by Lemma 5.6 (i)-(iii) together with Remark 5.9, we obtain for the states $\omega(\cdot)=\omega_{\rho_{k}}(\cdot)$ the representation of (5.38) in the form:

$$
C_{k}(\theta)=\omega_{\rho_{k}}(\widehat{w}(\theta))=\exp \left[-\frac{1}{4} \omega_{\rho_{k}}^{T}\left(\Phi^{2}(\theta)\right)+R(\theta)\right], k=1,2, \ldots, N,
$$

where $R(\theta)=O\left(|\theta|^{3}\right)$ in the vicinity of $\theta=0$. For the self-adjoint operator $\Phi(\theta)=\bar{\theta} a+\theta a^{*}$, the hypothesis (h2) and Lemma 5.6 (i) imply

$$
\omega_{\rho_{k}}^{T}\left(\Phi^{2}(\theta)\right)=|\theta|^{2} \operatorname{Tr}_{\mathscr{F}}\left[\rho_{k}\left(a^{*} a+a a^{*}\right)\right] .
$$

Now, taking into account Lemma 2.2 for the vector $\zeta_{\theta}$, as well as (5.39) and (5.40), we obtain the representation:

$$
\begin{gathered}
\omega_{\mathcal{S}}^{N \tau}(\widehat{w}(\theta))=\omega_{\rho(N \tau)}\left(W\left(\zeta_{\theta}\right)\right)=C_{0}\left(e^{i \epsilon \tau N}(g z)^{N} \theta\right) \prod_{k=1}^{N} C_{k}\left(e^{i \epsilon \tau N} g w(g z)^{N-k} \theta\right) \\
=C_{0}\left(e^{i \epsilon \tau N}(g z)^{N} \theta\right) \exp \left(-\sum_{k=1}^{N} \frac{\left|\theta_{k}\right|^{2}}{4} \operatorname{Tr}_{\mathscr{F}}\left[\left(a^{*} a+a a^{*}\right) \rho_{k}\right]+\widehat{R}\right)
\end{gathered}
$$

Here by (2.17) and by (5.39) one has

$$
\theta_{k}:=e^{i \epsilon N \tau} g w(g z)^{N-k} \theta, \sum_{k=1}^{N}\left|\theta_{k}\right|^{2}=|\theta|^{2}|w|^{2} \frac{1-|z|^{2 N}}{1-|z|^{2}}, \widehat{R}=\sum_{k=1}^{N} O\left(\left|\theta_{k}\right|^{3}\right) .
$$

By virtue of (1.12) and (1.13), we get $|g(\tau)|=1,|w(\tau)|^{2}+|z(\tau)|^{2}=1$ and also

$$
w(\tau)=i \eta \tau+O\left(\tau^{3}\right),|z(\tau)|=1-\frac{|\eta|^{2} \tau^{2}}{2}+O\left(\tau^{4}\right)
$$

for small $\tau$. This yields for small $\tau>0$ and large $N$, the estimates $\left|(g z)^{N}\right| \leq O\left(e^{-|\eta|^{2} \tau^{2} N / 2}\right)$, $\left|\theta_{k}\right| \leq O(\tau)$, and $\widehat{R}=O\left(\tau^{3} N\right)$ by virtue of (h1). Then taking into account the conditions $\tau^{2} N \rightarrow \infty$ and $\tau^{3} N \rightarrow 0$, we get the limits:

$$
\lim _{\tau \rightarrow 0, N \rightarrow \infty} C_{0}\left(e^{i \epsilon \tau N}(g z)^{N} \theta\right)=1, \lim _{\tau \rightarrow 0, N \rightarrow \infty} \sum_{k=1}^{N}\left|\theta_{k}\right|^{2}=|\theta|^{2}, \lim _{\tau \rightarrow 0, N \rightarrow \infty} \widehat{R}=0 .
$$

$C_{0}$ is a continuous function since it is defined by a normal state with density matrix $\rho_{0}$. Inserting all these limits into (5.41), we obtain what is claimed as the limit (5.36).

Corollary 5.10 Suppose that density matrices $\left\{\rho_{k}\right\}_{k=1}^{N}$ correspond to the gauge-invariant quasi-free Gibbs state with parameter $\beta$ (4.1). These states satisfy (h1)-(h3). The statement of Theorem 5.7 is valid with the limit

$$
\lim _{\tau \rightarrow 0, N \rightarrow \infty} \omega_{\mathcal{S}}^{N \tau}(\widehat{w}(\theta))=\exp \left\{-\frac{|\theta|^{2}}{4} \frac{1+e^{-\beta}}{1-e^{-\beta}}\right\}
$$

It coincides with the result for equilibrium state (5.6) of the subsystem $\mathcal{S}$. 
Hence, the short-time perturbation limit $\tau \rightarrow 0, N \rightarrow \infty$ subjected to $\tau^{2} N \rightarrow \infty$ and $\tau^{3} N \rightarrow 0$ gives a universal gauge-invariant quasi-free limiting state under hypothesis (h1)-(h3). The hypotheses (h2),(h3) control only first two moments of the initial states of the subsystem $\mathcal{C}$. Then stationarity and independence of repeated perturbation due to (h1), correspond to conditions for the non-commutative Central Limit Theorem [Ve]. Note also that the state $\omega_{\rho_{0}}$ of the subsystem $\mathcal{S}$ may be replaced by any regular state.

\section{Acknowledgements}

H.T. thanks JSPS for the financial support under the Grant-in-Aid for Scientific Research (C) 24540168. V.A.Z. is thankful to the Institute of Science and Engineering of Kanazawa University for support and hospitality.

\section{References}

[Ar1] H.Araki, Relative Entropy of States of von Neumann Algebras, Publ. RIMS, Kyoto Univ., 11 (1976), 809-833.

[AJP1] Open Quantum Systems I, The Hamiltonian Approach, S. Attal, A. Joye, C.A. Pillet (Eds.), Lecture Notes in Mathematics 1880, Springer-Verlag, BerlinHeidelberg 2006.

[AJPII] Open Quantum Systems II, The Markovian Approach, S. Attal, A. Joye, C.A. Pillet (Eds.), Lecture Notes in Mathematics 1881, Springer-Verlag, BerlinHeidelberg 2006.

[AJP3] Open Quantum Systems III, Recent Developements, S. Attal, A. Joye, C.A. Pillet (Eds.), Lecture Notes in Mathematics 1882, Springer-Verlag, BerlinHeidelberg 2006.

[BJM] L.Bruneau, A.Joye, and M.Merkli, Repeated interactions in open quantum systems, J.Math.Phys. 55 (2014), 075204.

[BR2] O. Bratteli and D.W. Robinson, Operator Algebras and Quantum Statistical Mechanics,vol.2, Springer-Verlag (2nd Edt), Berlin 1997.

[Fa] M.Fannes, The entropy of quasi-free states for a continuous boson system, Ann.de l'IHP, section A, 28(1978) 187-196.

[NVZ] B. Nachtergaele, A. Vershynina, and V. A. Zagrebnov, Non-Equilibrium States of a Photon Cavity Pumped by an Atomic Beam, Annales Henri Poincaré, 15 (2014), 213-262.

[TZ] H.Tamura and V. A. Zagrebnov, A Dynamics Driven by Repeaded Harmonic Perturbations, arXiv:1404.2998 (2014), 1-40.

[Ve] A.F. Verbeure, Many-Body Boson Systems, Springer-Verlag, Berlin 2011.

[Za] V.A. Zagrebnov, Topics in the Theory of Gibbs Semigroups, KU Leuven University Press, Leuven 2003. 